\title{
Orthogonal Synthesis of Covalent Polydendrimer Frameworks by Fusing Classical and Onion-peel Phosphorus-based dendritic Units
}

Nadia Katir, Nabil El Brahmi, Nathalie Marcotte, Jean Pierre Majoral, Mosto Bousmina, Abdelkrim El Kadib

S1. Synthesis of dendrimers D- $\mathrm{CHO}$, D- ${ }_{\mathrm{NNH} 2}$, D- $\mathrm{COOH}$ and Vio- $\mathrm{CHO}$

S2. DRIFT spectra

S3. ${ }^{13}$ C CP MAS NMR spectra

S4. ${ }^{31} P$ CP MAS NMR spectra

S5. DRUV spectra

S6. SEM analysis

S7. Nitrogen sorption analysis

S8. DRX analysis

S9. TEM analysis 
Synthesis of 1-CHO, 2-CHO and 3-CHO ${ }^{[1]}$ : Reaction of hexachlorocyclotriphosphazene core with hydroxybenzaldehyde in basic conditions affords the 1-сно. Condensation of the aldehyde groups with a phosphorhydrazides affords dendrimer of first generation denoted as a G1. Nucleophilic substitution reaction between $\mathbf{G}_{1}$ and hydroxybenzaldehyde affords 2 -сно. The repetition of the above mentioned steps was carried out until the obtaining of $\underline{\mathbf{3 - C H O}}$.

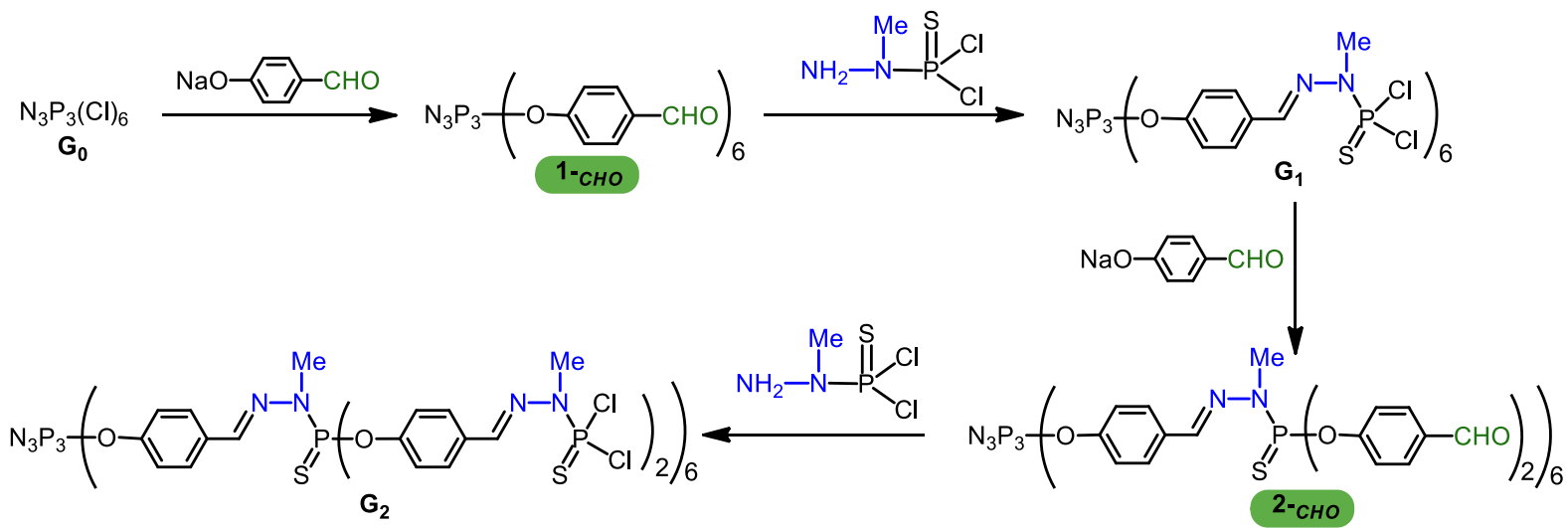

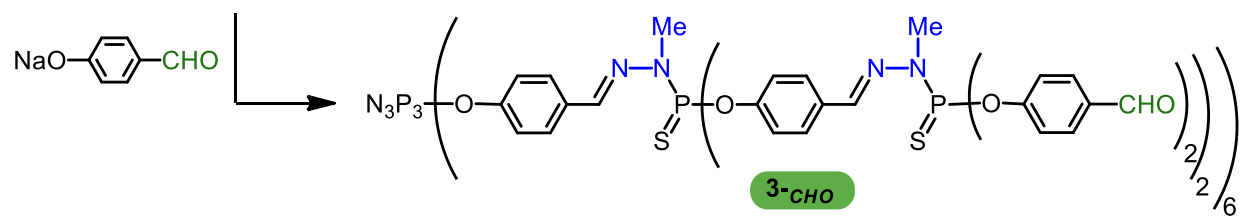

Dendrimer 1- $\mathrm{CHO}$ :

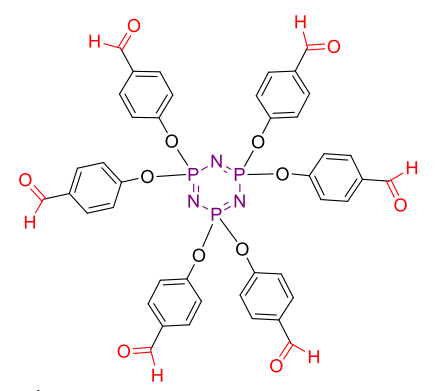

${ }^{31} \mathrm{P}\left\{{ }^{1} \mathrm{H}\right\}$ NMR $\left(122 \mathrm{MHz}, \mathrm{CDCl}_{3}\right) \delta: 7.07 ;{ }^{1} \mathrm{H}$ NMR $\left(300 \mathrm{MHz}, \mathrm{CDCl}_{3}\right) \delta 7.16(\mathrm{~d}, J=8.5 \mathrm{~Hz}, 12 \mathrm{H}, \mathrm{CH}), 7.75(\mathrm{~d}$, $J=8.6 \mathrm{~Hz}, 12 \mathrm{H}, \mathrm{CH}), 9.95(\mathrm{~s}, 6 \mathrm{H}, \mathrm{CHO}) ;{ }^{13} \mathrm{C}\left\{{ }^{1} \mathrm{H}\right\} \mathrm{NMR}\left(101 \mathrm{MHz}, \mathrm{CDCl}_{3}\right) \delta 121.20\left(\mathrm{dd}, J=3.5,1.8 \mathrm{~Hz}_{,} \mathrm{C}_{6} \mathrm{H}_{4}\right)$, 131.37, $133.76\left(\mathrm{C}_{6} \mathrm{H}_{4}\right), 154.46\left(\mathrm{dd}, J=5.0,2.5 \mathrm{~Hz}, \mathrm{C}_{6} \mathrm{H}_{4}\right), 190.38\left(\mathrm{C}_{6} \mathrm{H}_{4}\right)$.

\section{Dendrimer 2-Сно :}

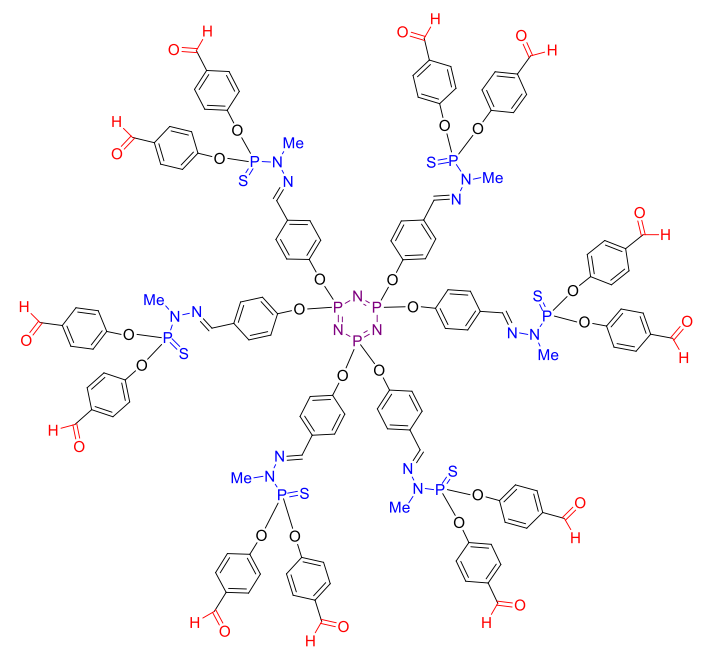


${ }^{31} \mathrm{P}\left\{{ }^{1} \mathrm{H}\right\} \mathrm{NMR}\left(122 \mathrm{MHz}, \mathrm{CDCl}_{3}\right) \delta 7.97\left(\mathrm{~N}_{3} \mathrm{P}_{3}\right), 60.40(\mathrm{P}=\mathrm{S}) ;{ }^{1} \mathrm{H}$ NMR $\left(300 \mathrm{MHz}, \mathrm{CDCl}_{3}\right) \delta 3.34(\mathrm{~d}, J=10.6$ $\left.\mathrm{Hz}, 18 \mathrm{H}, \mathrm{N}-\mathrm{CH}_{3}\right), 7.04(\mathrm{~d}, J=8.6 \mathrm{~Hz}, 14 \mathrm{H}, \mathrm{CH}), 7.34(\mathrm{dd}, J=8.5,1.5 \mathrm{~Hz}, 24 \mathrm{H}, \mathrm{CH}), 7.59(\mathrm{~d}, J=8.7 \mathrm{~Hz}, 12 \mathrm{H}$, $\mathrm{CH}), 7.64(\mathrm{~d}, J=2.1 \mathrm{~Hz}, 6 \mathrm{H}, \mathrm{CH}=\mathrm{N}), 7.86-7.78(\mathrm{~m}, 24 \mathrm{H}, \mathrm{CH}), 9.92(\mathrm{~s}, 6 \mathrm{H}, \mathrm{CHO}) ;{ }^{13} \mathrm{C}\left\{{ }^{1} \mathrm{H}\right\} \mathrm{NMR}(101 \mathrm{MHz}$, $\left.\mathrm{CDCl}_{3}\right) \delta 32.91\left(\mathrm{~d}, J=12.6 \mathrm{~Hz}, \mathrm{~N}-\mathrm{CH}_{3}\right), 121.39\left(\mathrm{~d}, J=3.0 \mathrm{~Hz}, \mathrm{C}_{6} \mathrm{H}_{4}\right),, 121.90\left(\mathrm{~d}, J=4.8 \mathrm{~Hz}, \mathrm{C}_{6} \mathrm{H}_{4}\right), 128.29$ $\left(\mathrm{C}_{6} \mathrm{H}_{4}\right), 131.43\left(\mathrm{C}_{6} \mathrm{H}_{4}\right), 131.82\left(\mathrm{C}_{6} \mathrm{H}_{4}\right), 133.72\left(\mathrm{~d}, J=1.4 \mathrm{~Hz}, \mathrm{C}_{6} \mathrm{H}_{4}\right), 139.36(\mathrm{~d}, J=14.4 \mathrm{~Hz}, \mathrm{CH}=\mathrm{N}), 155.00(\mathrm{~d}, J$ $\left.=7.0 \mathrm{~Hz}, \mathrm{C}_{6} \mathrm{H}_{4}\right), 151.46\left(\mathrm{dd}, J=5.3,2.6 \mathrm{~Hz}, \mathrm{C}_{6} \mathrm{H}_{4}\right), 190.59(\mathrm{CHO})$.

Dendrimer 3-Сно :

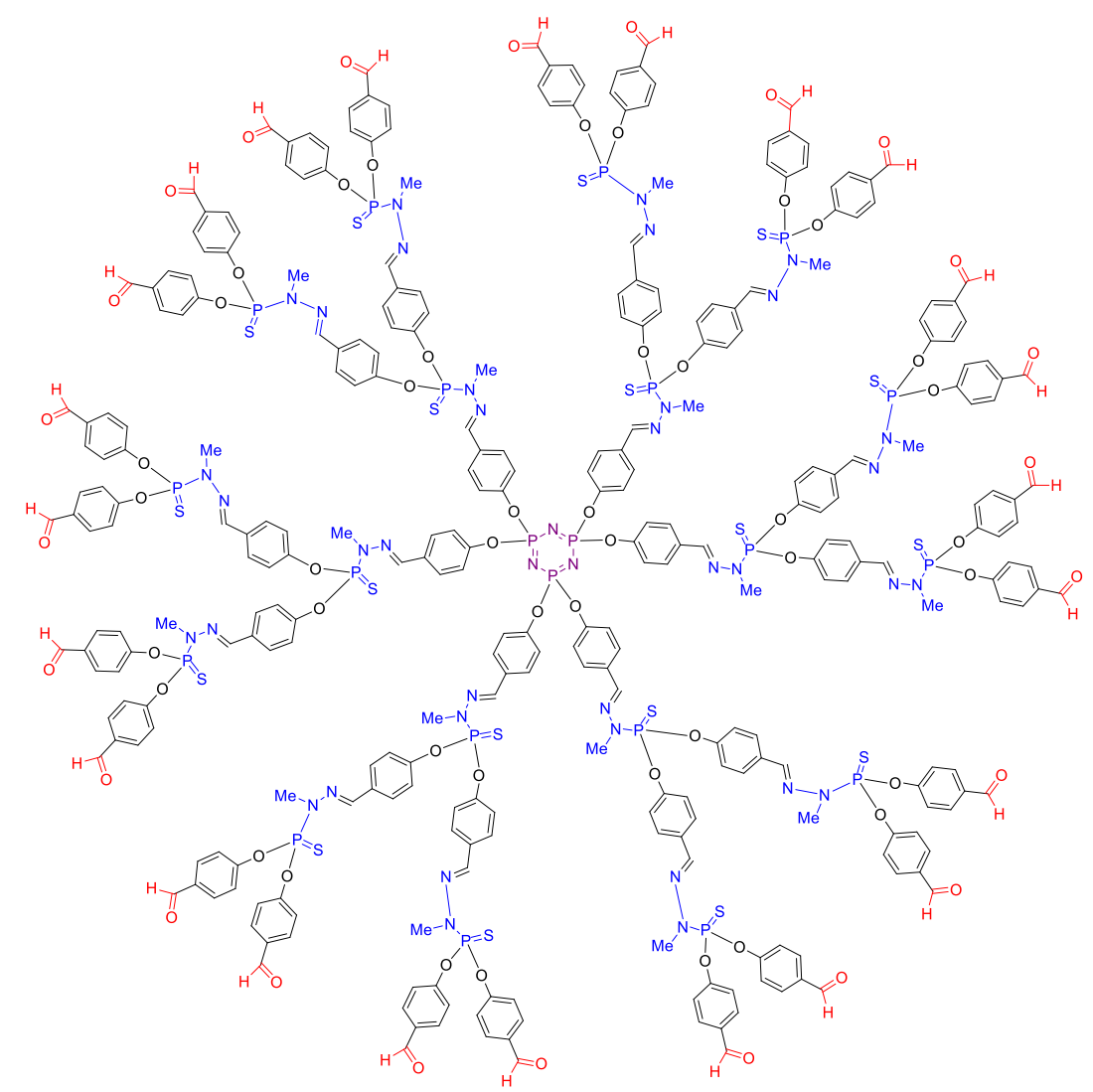

${ }^{31} \mathrm{P}\left\{{ }^{1} \mathrm{H}\right\}$ NMR $\left(122 \mathrm{MHz}, \mathrm{CDCl}_{3}\right) \delta 8.21\left(\mathrm{~N}_{3} \mathrm{P}_{3}\right), 60.26(\mathrm{P}=\mathrm{S}), 62.25(\mathrm{P}=\mathrm{S}),{ }^{1} \mathrm{H}$ NMR $\left(300 \mathrm{MHz}, \mathrm{CDCl}_{3}\right) \delta 3.26$ $\left(\mathrm{d}, J=10.2 \mathrm{~Hz}, 18 \mathrm{H}, \mathrm{N}-\mathrm{CH}_{3}\right), 3.36\left(\mathrm{~d}, J=10.8 \mathrm{~Hz}, 36 \mathrm{H}, \mathrm{N}-\mathrm{CH}_{3}\right), 6.94(\mathrm{~d}, J=8.4 \mathrm{~Hz}, 12 \mathrm{H}, \mathrm{CH}), 7.19(\mathrm{~d}, J=8.3$ $\mathrm{Hz}, 24 \mathrm{H}, \mathrm{CH}), 7.36-7.30(\mathrm{~m}, 48 \mathrm{H}, \mathrm{CH}), 7.63-7.57(\mathrm{~m}, 48 \mathrm{H}, \mathrm{CH}), 7.66-7.63(\mathrm{~m}, 18 \mathrm{H}, \mathrm{CH}=\mathrm{N}), 7.81(\mathrm{~d}, J=$ $8.3 \mathrm{~Hz}, 48 \mathrm{H}, \mathrm{CH}), 9.90$ (s, 24H, CHO). ${ }^{13} \mathrm{C}\left\{{ }^{1} \mathrm{H}\right\} \mathrm{NMR}\left(101 \mathrm{MHz}, \mathrm{CDCl}_{3}\right) \delta 32.92\left(\mathrm{~d}, J=12.8 \mathrm{~Hz}, \mathrm{~N}-\mathrm{CH}_{3}\right), 121.27$ $\left(\mathrm{C}_{6} \mathrm{H}_{4}\right), 121.77\left(\mathrm{~d}, J=4.8 \mathrm{~Hz}, \mathrm{C}_{6} \mathrm{H}_{4}\right), 121.94\left(\mathrm{~d}, J=4.7 \mathrm{~Hz}, \mathrm{C}_{6} \mathrm{H}_{4}\right), 128.33\left(\mathrm{C}_{6} \mathrm{H}_{4}\right), 131.43\left(\mathrm{C}_{6} \mathrm{H}_{4}\right), 131.96\left(\mathrm{C}_{6} \mathrm{H}_{4}\right)$, $132.08\left(\mathrm{C}_{6} \mathrm{H}_{4}\right), 133.65\left(\mathrm{C}_{6} \mathrm{H}_{4}\right), 139.05(\mathrm{~d}, J=14.0 \mathrm{~Hz}, \mathrm{CH}=\mathrm{N}), 139.48(\mathrm{~d}, J=13.8 \mathrm{~Hz}, \mathrm{CH}=\mathrm{N}), 151.20\left(\mathrm{C}_{6} \mathrm{H}_{4}\right)$, $151.43\left(\mathrm{~d}, J=7.1 \mathrm{~Hz}, \mathrm{C}_{6} \mathrm{H}_{4}\right), 155.06$ (d, $\left.J=7.0 \mathrm{~Hz}, \mathrm{C}_{6} \mathrm{H}_{4}\right), 190.67(\mathrm{CHO})$.

Synthesis of 1- ${ }^{N} \mathrm{H}_{2}, 2-{ }^{-} \mathrm{NH}_{2}$ and 3- ${ }^{-1 \mathrm{H}_{2}}{ }^{[2]}$ :

Synthesis of 1-NNH2

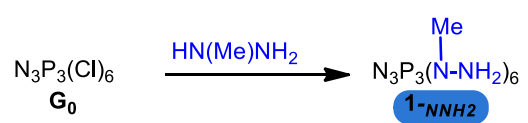

To a solution of methylhydrazine $(5.1 \mathrm{~mL}, 96 \mathrm{mmol})$ in $40 \mathrm{~mL}$ of chloroform was added dropwise a solution of hexachlorocyclotriphosphazene $(2.8 \mathrm{~g}, 8 \mathrm{mmol})$ in $15 \mathrm{~mL}$ of chloroform at $0^{\circ} \mathrm{C}$. The mixture was stirred for 10 hours at room temperature and then filtered through celite. The solvent was evaporated, and the residue was washed several times with a light $n$-pentane/chloroform (3/1) solution. 88\% yield. ${ }^{31} \mathrm{P}\left\{{ }^{1} \mathrm{H}\right\} \mathrm{NMR}\left(122 \mathrm{MHz}, \mathrm{CDCl}_{3}\right) \delta$ : 29.5; ${ }^{1} \mathrm{H}$ NMR $\left(300 \mathrm{MHz}, \mathrm{CDCl}_{3}\right) \delta 2.6$ (brs, $\left.18 \mathrm{H}, \mathrm{N}-\mathrm{CH}_{3}\right), 3.7$ (brs, $\left.12 \mathrm{H}, \mathrm{NH}\right) ;{ }^{13} \mathrm{C}\left\{{ }^{1} \mathrm{H}\right\} \mathrm{NMR}\left(101 \mathrm{MHz}, \mathrm{CDCl}_{3}\right)$ $\delta 39.5\left(\mathrm{~N}-\mathrm{CH}_{3}\right)$. 
Synthesis of 2-NNH2

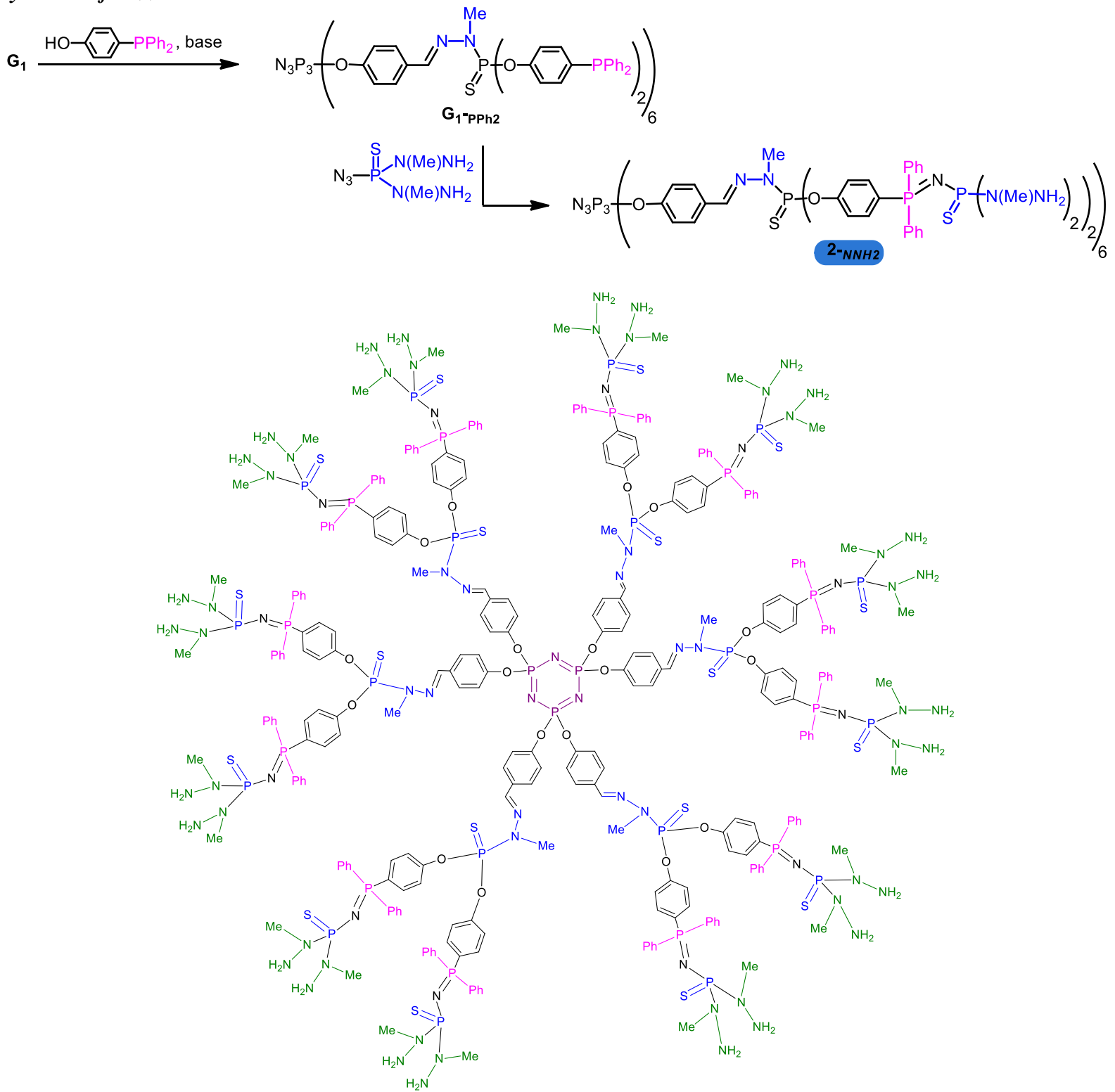

Cesium carbonate $\left(500 \mathrm{mg}, 1.3010^{-3} \mathrm{~mol}\right)$ was added to a solution of 4-(diphenylphosphino)phenol (190 mg, 0.68 $\left.10^{-3} \mathrm{~mol}\right)$ and $\mathbf{G 1}\left(100 \mathrm{mg}, 0.5310^{-4} \mathrm{mmol}\right)$ in THF $(10 \mathrm{~mL})$. The mixture was stirred overnight at room temperature, then filtered under argon and the filtrate was evaporated to dryness. The resulting oil was dissolved in $\mathrm{CH}_{2} \mathrm{Cl}_{2}(1 \mathrm{~mL})$ and this solution was added to a mixture of $50 \mathrm{~mL}$ of $n$-pentane/ $/ \mathrm{Et}_{2} \mathrm{O}(8 / 1)$ to allow dendrimer G1-pph2 to precipitate. To a mixture of dendrimer G1-PPh2 $\left(150 \mathrm{mg}, 0.32 \quad 10^{-3} \mathrm{~mol}\right)$ and azidothiobishydrazinophosphine ( $85 \mathrm{mg}, 0.43510^{-3} \mathrm{~mol}$ ) was added a minimum amount of $\mathrm{CH}_{2} \mathrm{Cl}_{2}$ to solubilize both reagents $(4 \mathrm{~mL})$. The resulting solution was stirred at room temperature for 2 days (monitoring by ${ }^{31} \mathrm{P} \mathrm{NMR}$ ). The mixture was washed twice with $n$-pentane/ $\mathrm{CH}_{2} \mathrm{Cl}_{2}(10 / 3)$ to afford dendrimer 2- $\mathrm{NNH2}$ as a white powder in 85 $\%$ yield. ${ }^{31} \mathrm{P}\left\{{ }^{1} \mathrm{H}\right\}$ NMR $\left(162 \mathrm{MHz}, \mathrm{CD}_{2} \mathrm{Cl}_{2}\right) \delta 7.57\left(\mathrm{~s}, \mathrm{~N}_{3} \mathrm{P}_{3}\right), 12.19\left(\mathrm{~d},{ }^{2} J_{P P}=17.0 \mathrm{~Hz}, \mathrm{PPh}_{2}\right), 60.76(\mathrm{~s}, \mathrm{P}=\mathrm{S}), 70.85$ $\left(\mathrm{d},{ }^{2} J_{P P}=17.0 \mathrm{~Hz}, \mathrm{P}=\mathrm{S}\right) .{ }^{1} \mathrm{H}$ NMR $\left(400 \mathrm{MHz}, \mathrm{CD}_{2} \mathrm{Cl}_{2}\right) \delta 2.65\left(\mathrm{dd},{ }^{3} J_{P H}=11.8 \mathrm{~Hz},{ }^{5} J_{P H}=1.9 \mathrm{~Hz}, 72 \mathrm{H}, \mathrm{N}_{-} \mathrm{CH}_{3}\right)$, $3.35\left(\mathrm{~d},{ }^{3} J_{P H}=10.3 \mathrm{~Hz}, 18 \mathrm{H}, \mathrm{N}-\mathrm{CH}_{3}\right), 7.05(\mathrm{~d}, J=7.6 \mathrm{~Hz}, 12 \mathrm{H}, \mathrm{CH}), 7.42-7.52(\mathrm{~m}, 24 \mathrm{H}, \mathrm{CH}), 7.53-7.61(\mathrm{~m}$, $36 \mathrm{H}, \mathrm{CH}), 7.66-7.82\left(\mathrm{~m}, 120 \mathrm{H}, \mathrm{PPh}_{2}\right) .{ }^{13} \mathrm{C}\left\{{ }^{1} \mathrm{H}\right\}$ NMR $\left(101 \mathrm{MHz}, \mathrm{CD}_{2} \mathrm{Cl}_{2}\right) \delta 32.96\left(\mathrm{~s} . b r, \mathrm{~N}_{-} \mathrm{CH}_{3}\right), 39.34\left(\mathrm{~d},{ }^{2} J_{C P}\right.$ $\left.=7.5 \mathrm{~Hz}, \mathrm{~N}-\mathrm{CH}_{3}\right), 121.05\left(\mathrm{C}_{6} \mathrm{H}_{4}\right), 121.38\left(\mathrm{dd},{ }^{3} J_{C P}=7.1 \mathrm{~Hz},{ }^{3} J_{C P}=5.0 \mathrm{~Hz}, \mathrm{C}_{6} \mathrm{H}_{4}\right), 128.35\left(\mathrm{C}_{6} \mathrm{H}_{4}\right), 128.59\left(\mathrm{~d},{ }^{3} J_{C P}\right.$ $\left.=12.9 \mathrm{~Hz}, \mathrm{C}_{6} \mathrm{H}_{5}\right), 129.70\left(\mathrm{~d},{ }^{1} J_{C P}=106.9 \mathrm{~Hz}, \mathrm{C}_{6} \mathrm{H}_{4}\right), 131.84\left(\mathrm{~s}, \mathrm{C}_{6} \mathrm{H}_{4}\right), 132.34\left(\mathrm{~d},{ }^{l} J_{C P}=99.7 \mathrm{~Hz}, \mathrm{C}_{6} \mathrm{H}_{5}\right), 132.44$ $\left(\mathrm{s}, \mathrm{C}_{6} \mathrm{H}_{5}\right), 132.65\left(\mathrm{~d},{ }^{2} J_{C P}=10.7 \mathrm{~Hz}, \mathrm{C}_{6} \mathrm{H}_{5}\right), 134.75\left(\mathrm{~d},{ }^{2} J_{C P}=12.1 \mathrm{~Hz}, \mathrm{C}_{6} \mathrm{H}_{4}\right), 139.80-140.06(\mathrm{~m}, \mathrm{CH}=\mathrm{N}), 151.42$ $\left(\mathrm{s}, \mathrm{C}_{6} \mathrm{H}_{4}\right), 153.65\left(\mathrm{~s}, \mathrm{C}_{6} \mathrm{H}_{4}\right)$. 
Synthesis of 3- ${ }_{N N H}$
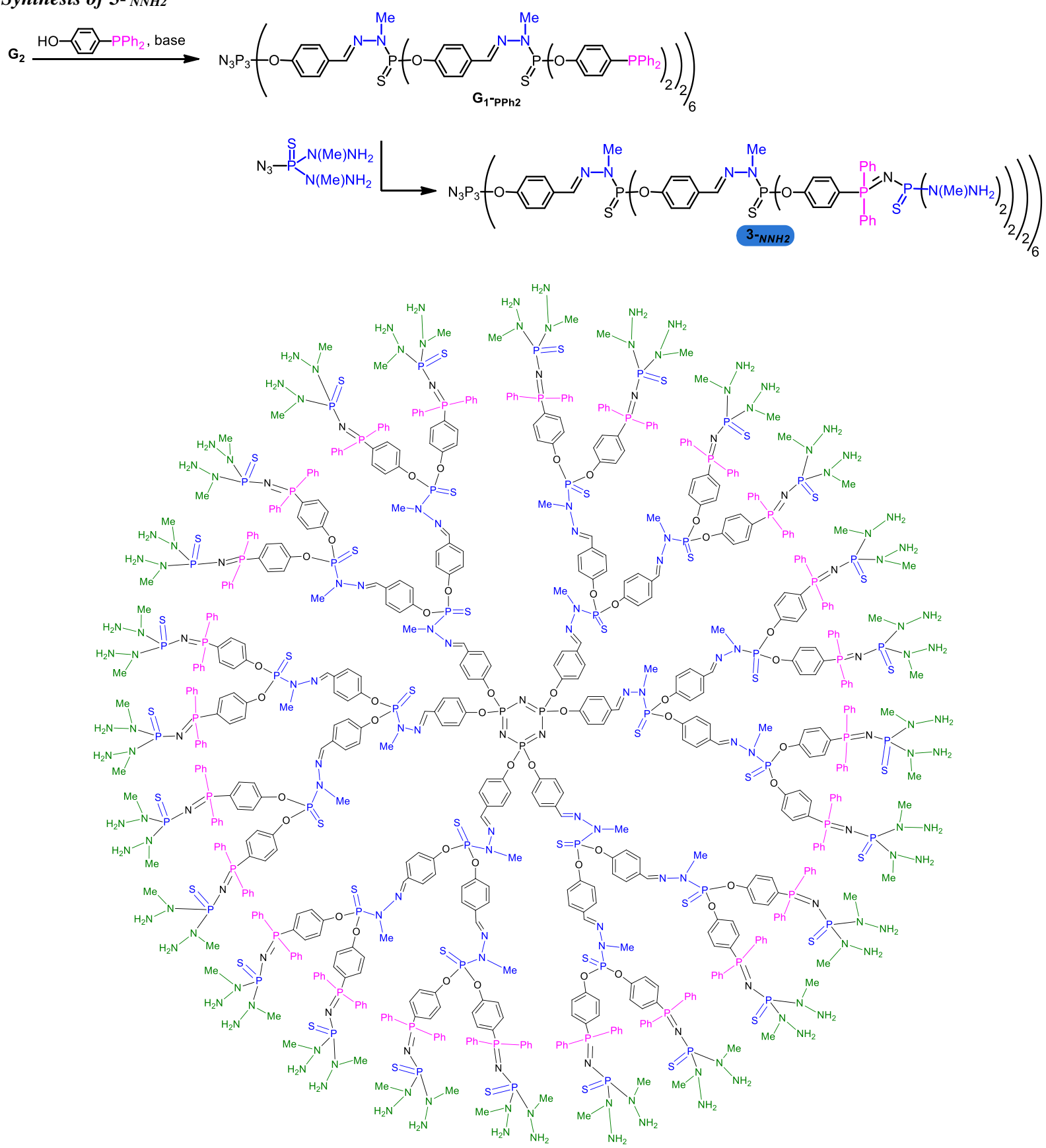

Cesium carbonate $\left(653 \mathrm{mg}, 2.0010^{-3} \mathrm{~mol}\right)$ was added to a solution of 4-(diphenylphosphino)phenol (290 mg, 1.04 $\mathrm{mmol})$ and G2 $\left(200 \mathrm{mg}, 0.4210^{-3} \mathrm{~mol}\right)$ in THF $(10 \mathrm{~mL})$. The mixture was stirred overnight at room temperature, then filtered and the filtrate was evaporated to dryness. The resulting oil was dissolved in $\mathrm{CH}_{2} \mathrm{Cl}_{2}(3 \mathrm{~mL})$ and this solution was added to a mixture of $100 \mathrm{~mL}$ of $n$-pentane/Et $2 \mathrm{O}(9 / 1)$ to allow dendrimer G2-pPh2 to precipitate. To a mixture of dendrimer G2-PPh2 $\left(150 \mathrm{mg}, 0.1410^{-3} \mathrm{~mol}\right)$ and azidothiobishydrazinophosphine (90 mg, $0.4610^{-3}$ mol) was added a minimum amount of $\mathrm{CH}_{2} \mathrm{Cl}_{2}$ to solubilize both reagents $(3 \mathrm{~mL})$. The resulting solution was stirred at room temperature for 4 days (monitoring by ${ }^{31} \mathrm{P} \mathrm{NMR}$ ). The mixture was washed twice with $n$ pentane $/ \mathrm{CH}_{2} \mathrm{Cl}_{2}(10 / 3)$ to afford dendrimer $\mathbf{G 2}-{ }_{N N H 2}$ as a white powder in $80 \%$ yield. ${ }^{31} \mathrm{P}\left\{{ }^{1} \mathrm{H}\right\} \mathrm{NMR}(162 \mathrm{MHz}$, $\left.\mathrm{CD}_{2} \mathrm{Cl}_{2}\right) \delta 8.09$ (s, $\mathrm{N}_{3} \mathrm{P}_{3}$ ), 12.30 (s.br, $\left.\mathrm{PPh}_{2}\right) 60.63$ (s, P=S), 62.49 (s, P=S), 70.72 (s.br, P=S). ${ }^{1} \mathrm{H}$ NMR $(400 \mathrm{MHz}$, $\left.\mathrm{CD}_{2} \mathrm{Cl}_{2}\right) \delta 2.65\left(\mathrm{~d},{ }^{3} J_{P H}=11.8 \mathrm{~Hz}, 144 \mathrm{H}, \mathrm{N}-\mathrm{CH}_{3}\right), 3.34\left(\mathrm{~d},{ }^{3} J_{P H}=9.4 \mathrm{~Hz}, 54 \mathrm{H}, \mathrm{N}-\mathrm{CH}_{3}\right), 7.15-7.79(\mathrm{~m}, 426 \mathrm{H}, \mathrm{CH}$, $\left.\mathrm{CH}=\mathrm{N}, \mathrm{PPh}_{2}\right) .{ }^{13} \mathrm{C}\left\{{ }^{1} \mathrm{H}\right\} \mathrm{NMR}\left(101 \mathrm{MHz}, \mathrm{CD}_{2} \mathrm{Cl}_{2}\right) \delta 32.97\left(\mathrm{~d},{ }^{2} J_{C P}=12.3 \mathrm{~Hz}, \mathrm{~N}-\mathrm{CH}_{3}\right), 39.33\left(\mathrm{~d},{ }^{2} J_{C P}=7.5 \mathrm{~Hz}, \mathrm{~N}-\right.$ $\left.\mathrm{CH}_{3}\right), 121.35\left(\mathrm{~d},{ }^{3} J_{C P}=7.3 \mathrm{~Hz}, \mathrm{C}_{6} \mathrm{H}_{4}\right), 121.48\left(\mathrm{~d},{ }^{3} J_{C P}=7.3 \mathrm{~Hz}, \mathrm{C}_{6} \mathrm{H}_{4}\right), 121.80\left(\mathrm{C}_{6} \mathrm{H}_{4}\right), 127.03\left(\mathrm{~d},{ }^{1} J_{C P}=105.9 \mathrm{~Hz}\right.$, $\left.\mathrm{C}_{6} \mathrm{H}_{4}\right), 128.37\left(\mathrm{C}_{6} \mathrm{H}_{4}\right), 128.60\left(\mathrm{~d},{ }^{3} J_{C P}=12.8 \mathrm{~Hz}, \mathrm{C}_{6} \mathrm{H}_{5}\right), 129.68\left(\mathrm{~d},{ }^{l} J_{C P}=106.8 \mathrm{~Hz}, \mathrm{C}_{6} \mathrm{H}_{5}\right), 132.45\left(\mathrm{C}_{6} \mathrm{H}_{5}\right), 132.67$ $\left(\mathrm{d},{ }^{2} J_{C P}=10.7 \mathrm{~Hz}, \mathrm{C}_{6} \mathrm{H}_{5}\right), 132.85\left(\mathrm{~s} . \mathrm{br}, \mathrm{C}_{6} \mathrm{H}_{4}\right), 134.72\left(\mathrm{~d},{ }^{2} J_{C P}=12.0 \mathrm{~Hz}, \mathrm{C}_{6} \mathrm{H}_{4}\right), 139.98(\mathrm{~s} . \mathrm{br}, \mathrm{CH}=\mathrm{N}), 151.39$ $\left(\mathrm{C}_{6} \mathrm{H}_{4}\right), 153.39$ (s.br, $\left.\mathrm{C}_{6} \mathrm{H}_{4}\right), 153.66$ (s.br, $\left.\mathrm{C}_{6} \mathrm{H}_{4}\right)$. 
Vio-сHо:

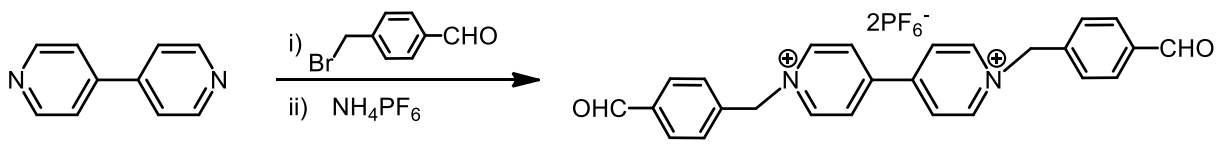

A solution of 4,4'-Bipyridine $(0.39 \mathrm{~g}, 2.50 \mathrm{mmol})$ and 4-(bromomethyl)benzaldehyde $(1.00 \mathrm{~g}, 4.97 \mathrm{mmol})$ in 15 $\mathrm{mL}$ of acetonitrile was heated at $75^{\circ} \mathrm{C}$ for $6 \mathrm{~h}$. The precipitate was filtered, washed with acetonitrile and ether, and dried in vacuo to yield $1.24 \mathrm{~g}(89 \%)$ of a yellow powder. The powder was dissolved in water, and $5 \mathrm{~mL}$ of a saturated aqueous solution of $\mathrm{NH}_{4} \mathrm{PF}_{6}$ was added dropwise. The resulting solid was filtered, washed with cold water and diethyl ether and dried under vacuum. mp $266-267^{\circ} \mathrm{C} .{ }^{1} \mathrm{H} \mathrm{NMR}\left(400 \mathrm{MHz}, \mathrm{CD}_{3} \mathrm{CN}\right): \delta 5.94(\mathrm{~s}, 4 \mathrm{H}$, $\left.\mathrm{CH}_{2}\right), 7.68\left(\mathrm{~d}, J=8.2 \mathrm{~Hz}, 4 \mathrm{H}, \mathrm{C}_{6} \mathrm{H}_{4}\right), 8.03\left(\mathrm{~d}, J=8.2 \mathrm{~Hz}, 4 \mathrm{H}, \mathrm{C}_{6} \mathrm{H}_{4}\right), 8.43\left(\mathrm{~d}, J=6.7 \mathrm{~Hz}, 4 \mathrm{H}, \mathrm{NC}_{5} \mathrm{H}_{4}\right), 9.01(\mathrm{~d}$, $\left.J=6.9 \mathrm{~Hz}, 4 \mathrm{H}, \mathrm{NC}_{5} \mathrm{H}_{4}\right), 10.07(\mathrm{~s}, 2 \mathrm{H}, \mathrm{CHO}) ;{ }^{13} \mathrm{C}\left\{{ }^{1} \mathrm{H}\right\} \mathrm{NMR}\left(101 \mathrm{MHz}, \mathrm{CD}_{3} \mathrm{CN}\right): \delta 64.12\left(\mathrm{CH}_{2}\right), 127.69\left(\mathrm{NC}_{5} \mathrm{H}_{4}\right)$, $129.84\left(\mathrm{C}_{6} \mathrm{H}_{4}\right), 130.32\left(\mathrm{C}_{6} \mathrm{H}_{4}\right), 137.45\left(\mathrm{C}_{6} \mathrm{H}_{4}\right), 138.46\left(\mathrm{C}_{6} \mathrm{H}_{4}\right), 145.90\left(\mathrm{NC}_{5} \mathrm{H}_{4}\right), 150.57\left(\mathrm{NC}_{5} \mathrm{H}_{4}\right), 192.10(\mathrm{CHO})$; ESI m/z 539.3 [M-PF $\left._{6}\right]^{+}$; IR (neat): $v 1698(\mathrm{C}=\mathrm{O}), 1639(\mathrm{C}=\mathrm{N}) \mathrm{cm}^{-1}$.

\section{Synthesis of 1- $\mathrm{COOH}$ and 2- $\mathrm{COOH}-[3]$ :}
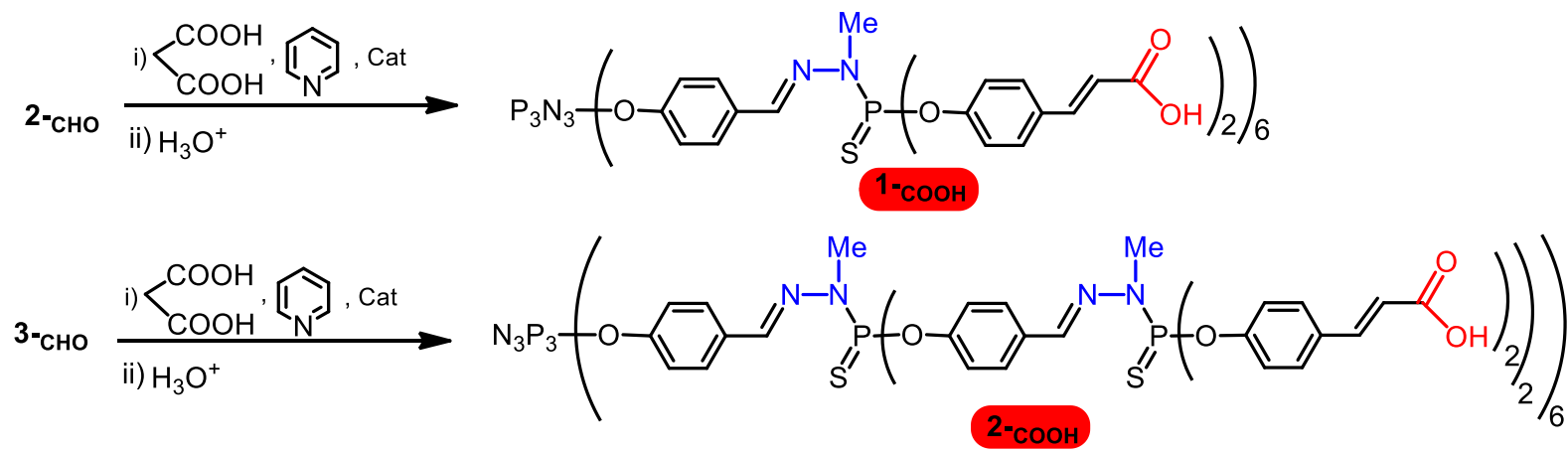

Malonic acid (excess) and piperidine (traces) were added to a solution of dendrimer (2-сно оr 3-сно) in pyridine. The solution was heated at $95^{\circ} \mathrm{C}$ with stirring for $15 \mathrm{~h}$, heated at reflux for $5 \mathrm{~min}$, cooled to $0^{\circ} \mathrm{C}$, and poured onto a mixture of crushed ice and hydrochloric acid. The precipitate was filtered off, washed with water, and dried under vacuum at room temperature. The resulting powder was dissolved in THF and precipitated with pentane to afford 1-СоОн or 2-соOн as a white powder.

\section{Dendrimer 1-COOH}

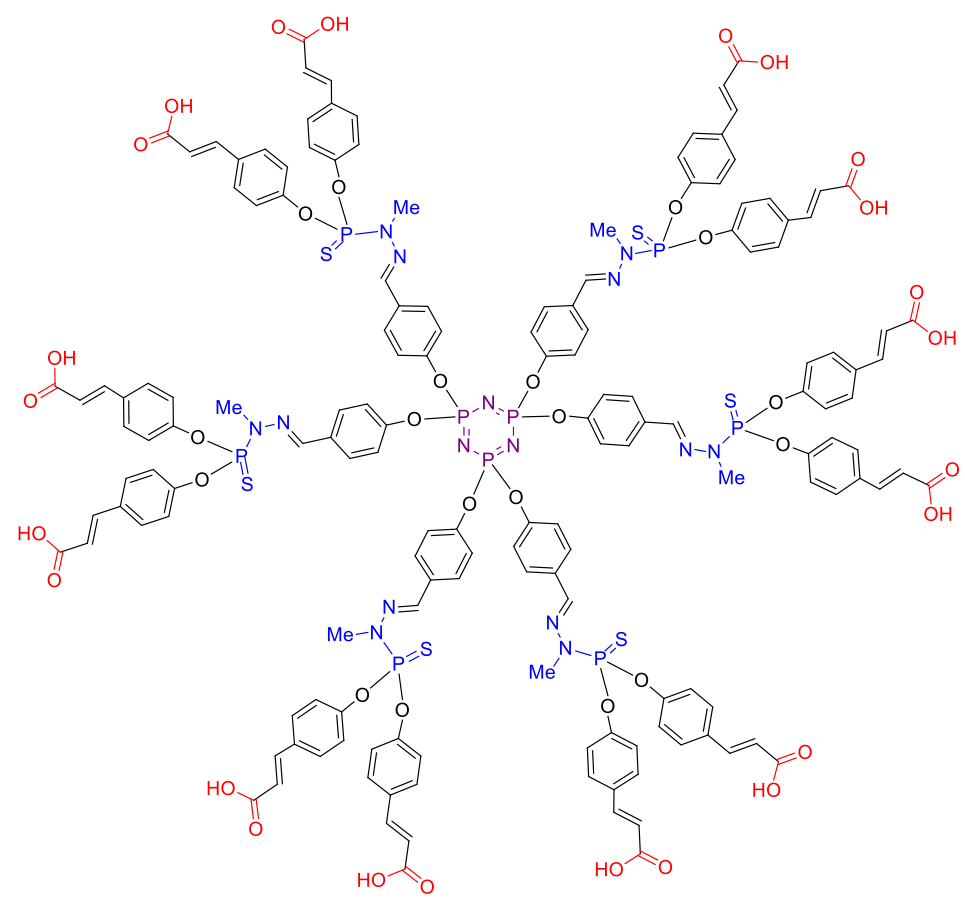


${ }^{1} \mathrm{H}$ NMR (DMSO, $\left.300 \mathrm{MHz}\right) \delta 3.32\left(\mathrm{~d}, J=9.6 \mathrm{~Hz}, 18 \mathrm{H} ; \mathrm{N}-\mathrm{CH}_{3}\right), 6.45(\mathrm{~d}, J=16.0 \mathrm{~Hz}, 12 \mathrm{H} ; \mathrm{Ph}-\mathrm{CH}), 7.05(\mathrm{~d}, J=$ $7.5 \mathrm{~Hz}, 12 \mathrm{H} ; \mathrm{CH}), 7.20(\mathrm{~d}, \mathrm{~J}=8.0 \mathrm{~Hz}, 24 \mathrm{H} ; \mathrm{CH}), 7.55$ (d, $J=16.0 \mathrm{~Hz}, 12 \mathrm{H} ; \mathrm{CH}-\mathrm{COOH}), 7.67$ (d, $J=8.0 \mathrm{~Hz}$, $36 \mathrm{H} ; \mathrm{CH}), 7.87(\mathrm{~s}, 6 \mathrm{H} ; \mathrm{CH}=\mathrm{N}) ;{ }^{13} \mathrm{C}\left\{{ }^{1} \mathrm{H}\right\}$ NMR (DMSO, $\left.75 \mathrm{MHz}\right) \delta 33.7\left(\mathrm{~d}, J=12.1 \mathrm{~Hz} ; \mathrm{N}_{-} \mathrm{CH}_{3}\right), 120.3(\mathrm{Ph}-$ $\mathrm{CH}), 121.9\left(\mathrm{C}_{6} \mathrm{H}_{4}\right), 122.2\left(\mathrm{C}_{6} \mathrm{H}_{4}\right), 129.2\left(\mathrm{C}_{6} \mathrm{H}_{4}\right), 130.7\left(\mathrm{C}_{6} \mathrm{H}_{4}\right), 132.6\left(\mathrm{C}_{6} \mathrm{H}_{4}\right), 132.9\left(\mathrm{C}_{6} \mathrm{H}_{4}\right), 141.4(\mathrm{~d}, \mathrm{~J}=13.2 \mathrm{~Hz}$; $\mathrm{CH}=\mathrm{N}$ ), 143.5 (s, CH-COOH), 151.4 (brs; $\left.\mathrm{C}_{6} \mathrm{H}_{4}\right), 152.0$ (d, J =6.6 Hz, $\left.\mathrm{C}_{6} \mathrm{H}_{4}\right), 168.4(\mathrm{COOH}) ;{ }^{31} \mathrm{P}\left\{{ }^{1} \mathrm{H}\right\} \mathrm{NMR}$ (DMSO, $121 \mathrm{MHz}) \delta 11.8\left(\mathrm{~s} ; \mathrm{N}_{3} \mathrm{P}_{3}\right), 65.4$ (s; $\mathrm{P}=\mathrm{S}$ ).

\section{Dendrimer 2-COOH}

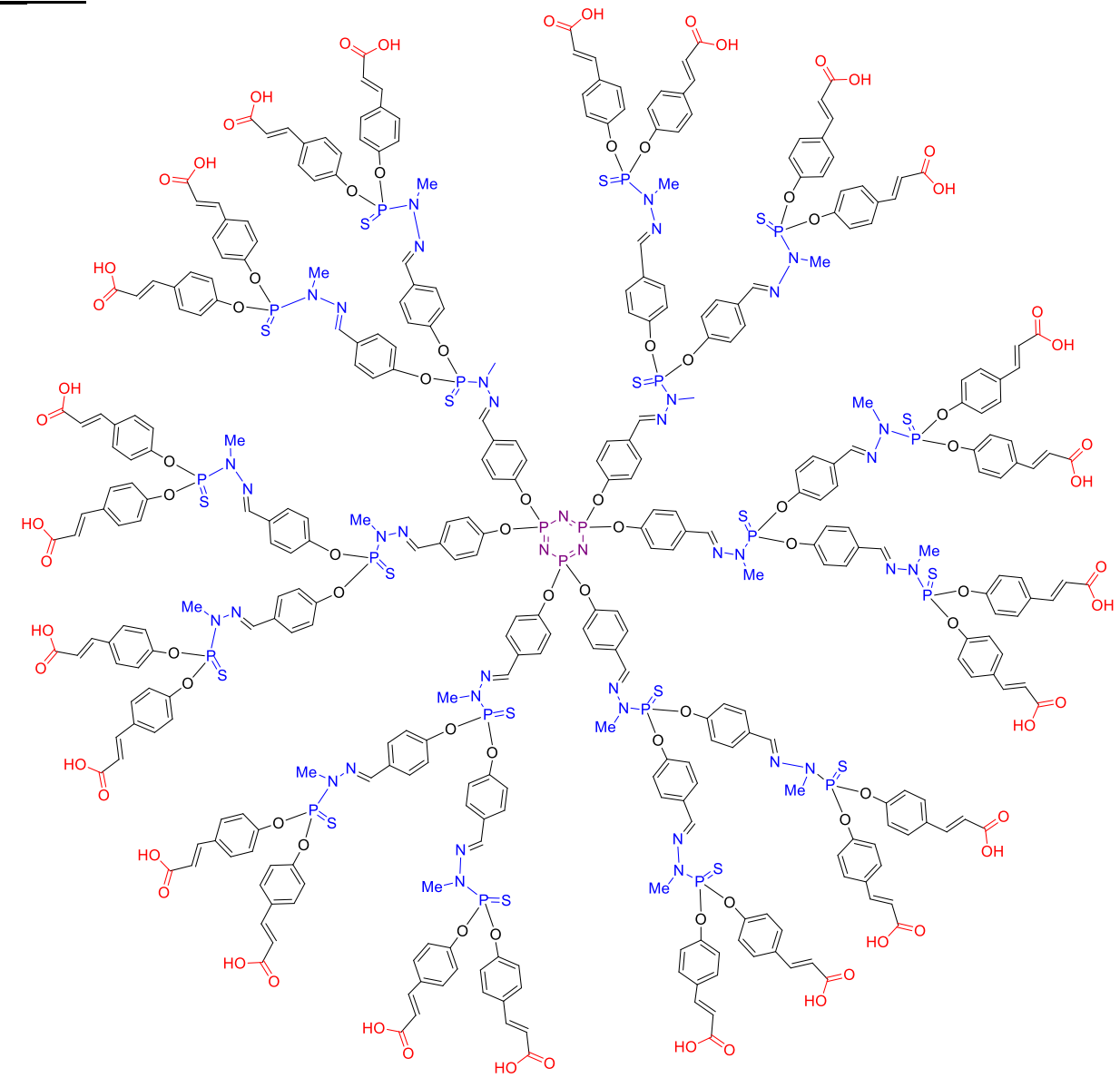

${ }^{13} \mathrm{C}\left\{{ }^{1} \mathrm{H}\right\}$ NMR $(100 \mathrm{MHz}) \delta 32.79\left(\mathrm{~N}-\mathrm{CH}_{3}\right), 118.76,121.84,131.08,151.46\left(\mathrm{C}_{6} \mathrm{H}_{4}\right), 170.37(\mathrm{COOH}) ;{ }^{31} \mathrm{P}\left\{{ }^{1} \mathrm{H}\right\}$ NMR (162 MHz) $\delta 9.09\left(\mathrm{~s} ; \mathrm{N}_{3} \mathrm{P}_{3}\right), 60.57(\mathrm{~s} ; \mathrm{P}=\mathrm{S})$.

[1] N. Launay, A. M. Caminade, R. Lahana, J. P. Majoral, Angew. Chem. Int. Ed. Engl. 1994, 33, 1589. [2] N. Katir, N. El Brahmi, A. El Kadib, S. Mignani, A.-M. Caminade, M. Bousmina, J. P. Majoral, Chem. Eur. J. 2015, 21, 6400.

[3] M. Blanzat, C.-O. Turrin, A.-M. Aubertin, C. Couturier-Vidal, A.-M. Caminade, J.-P. Majoral, I. RicoLattes, A. Lattes, ChemBioChem 2005, 6, 2207. 


\section{S2. DRIFT spectra analysis}
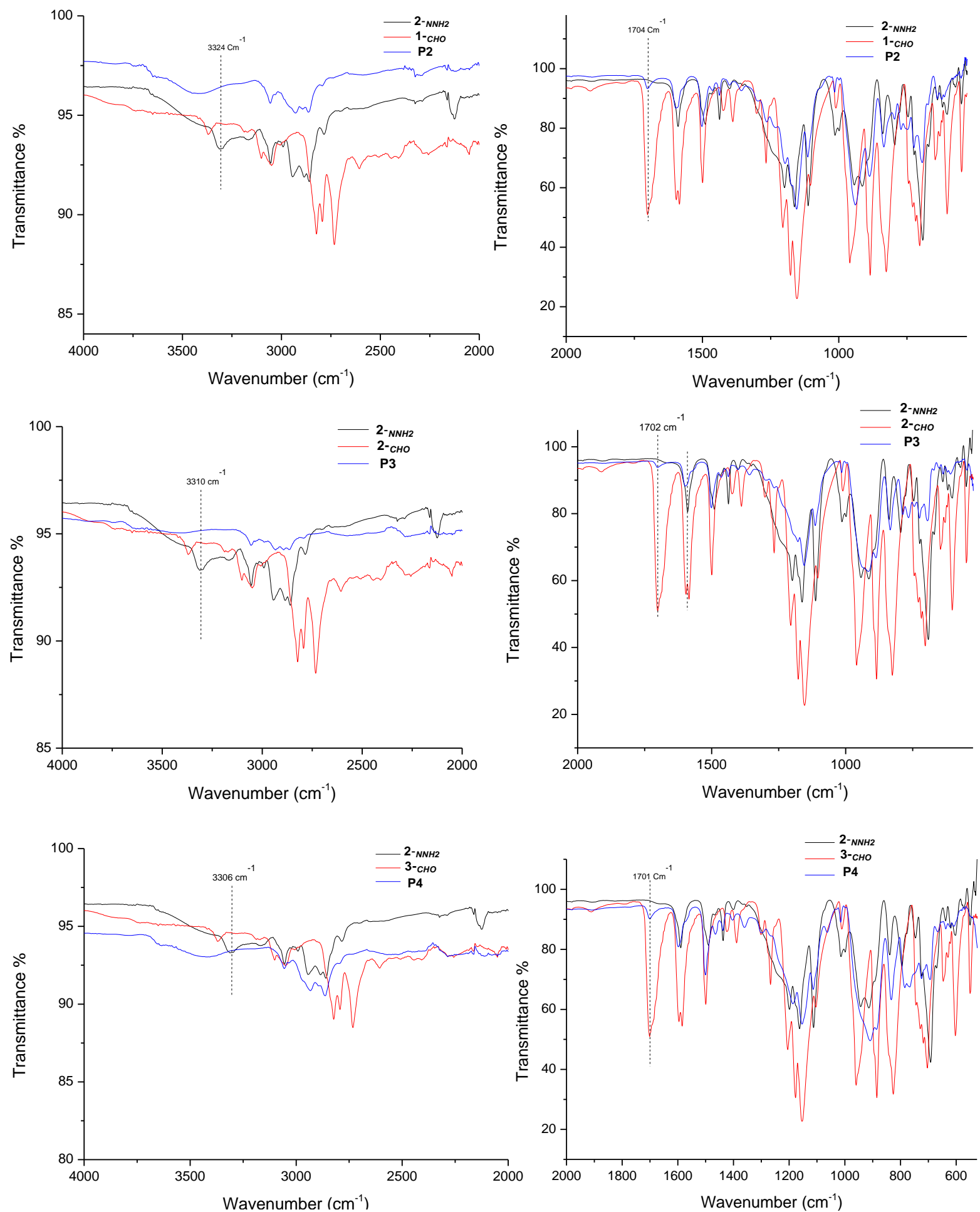

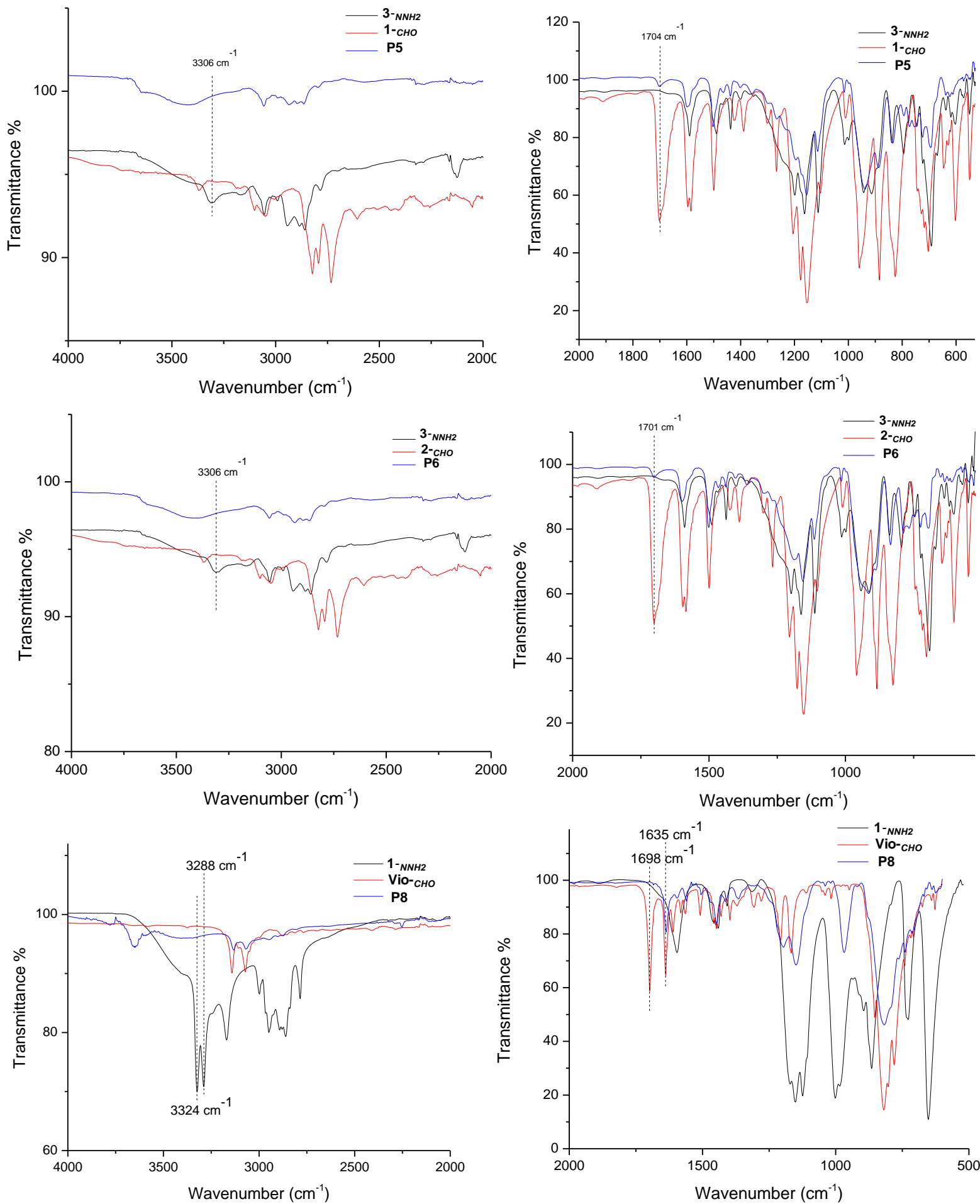

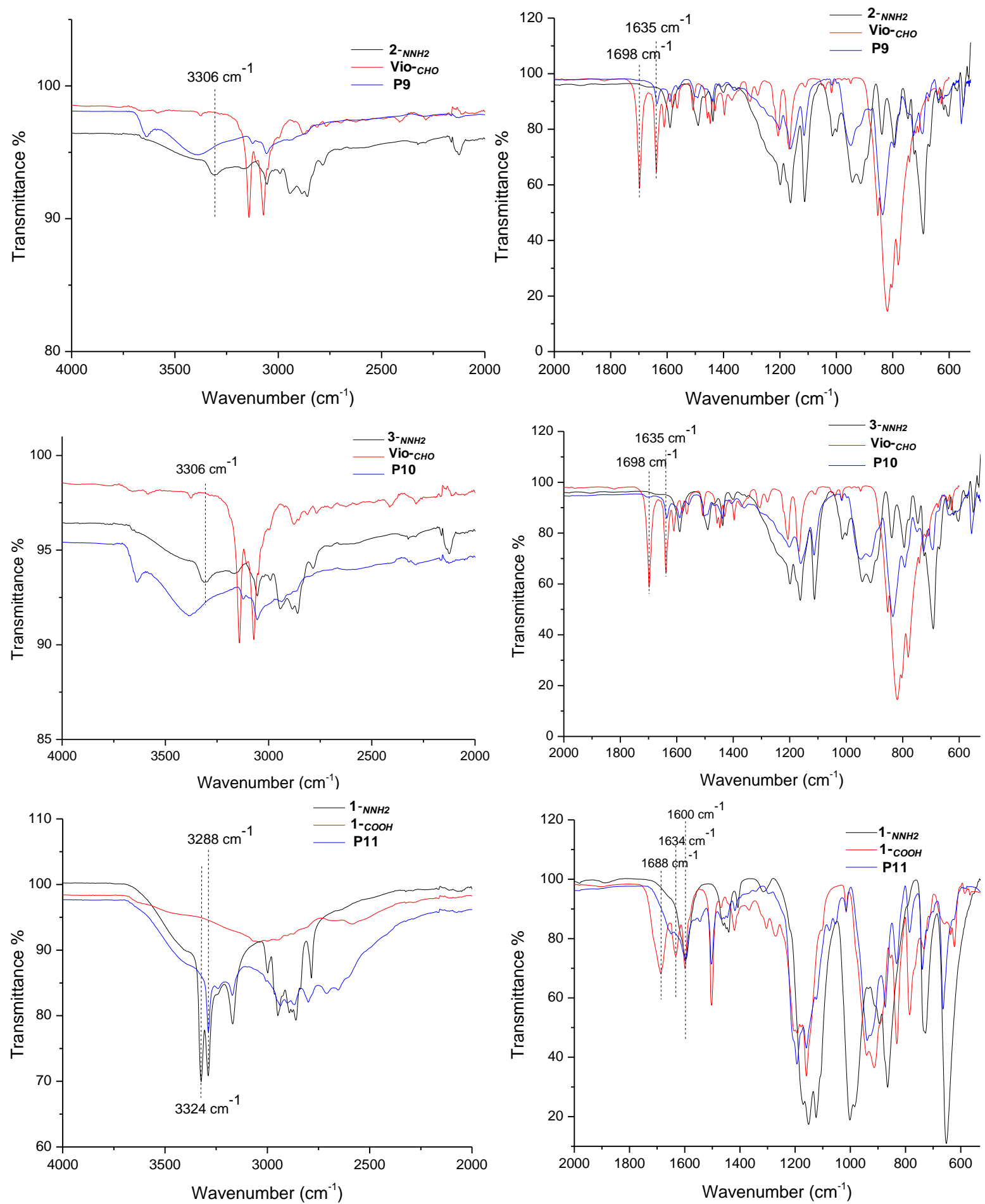

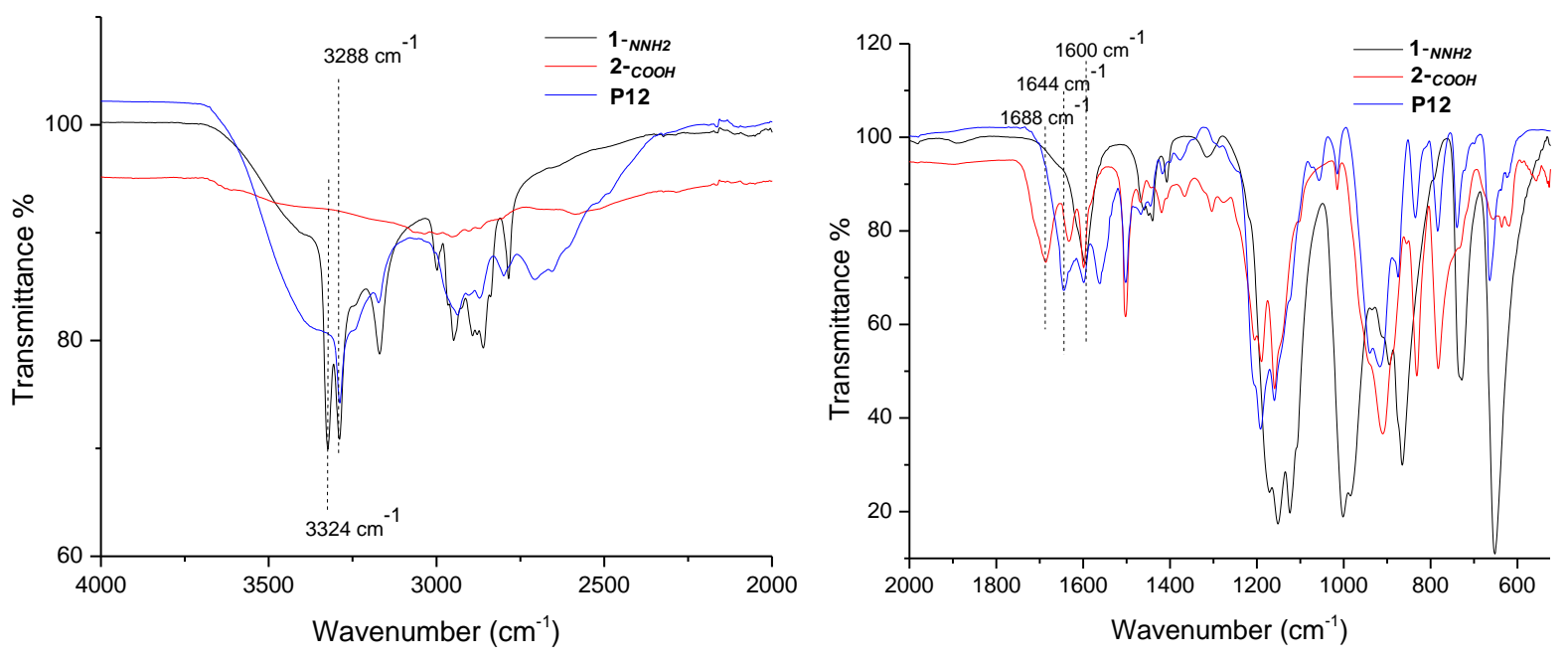
S3. ${ }^{13} \mathrm{C}$ CP MAS NMR spectra

${ }^{13} \mathrm{C}$ NMR of $P_{1}$

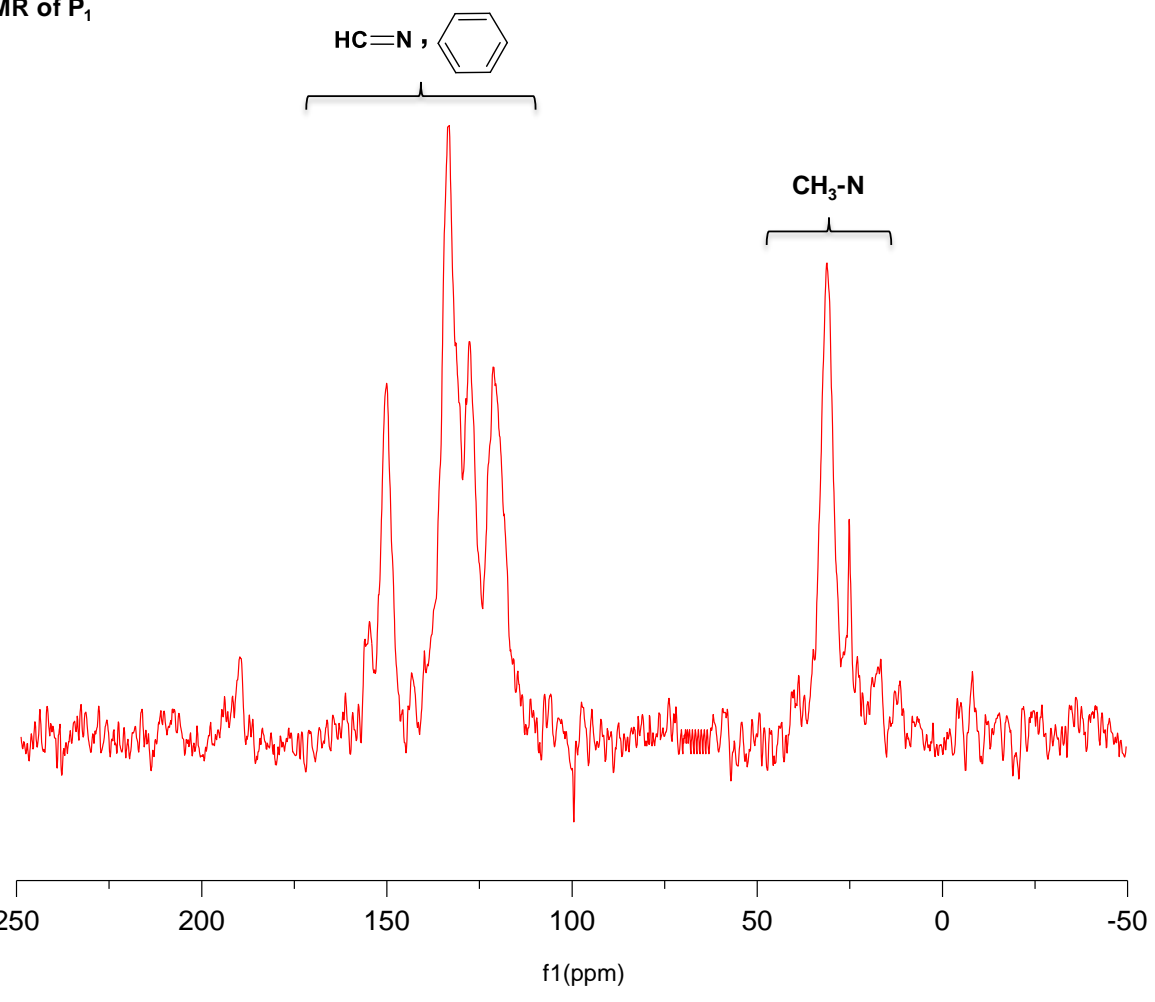

${ }^{13} \mathrm{C}$ NMR of $P_{3}$

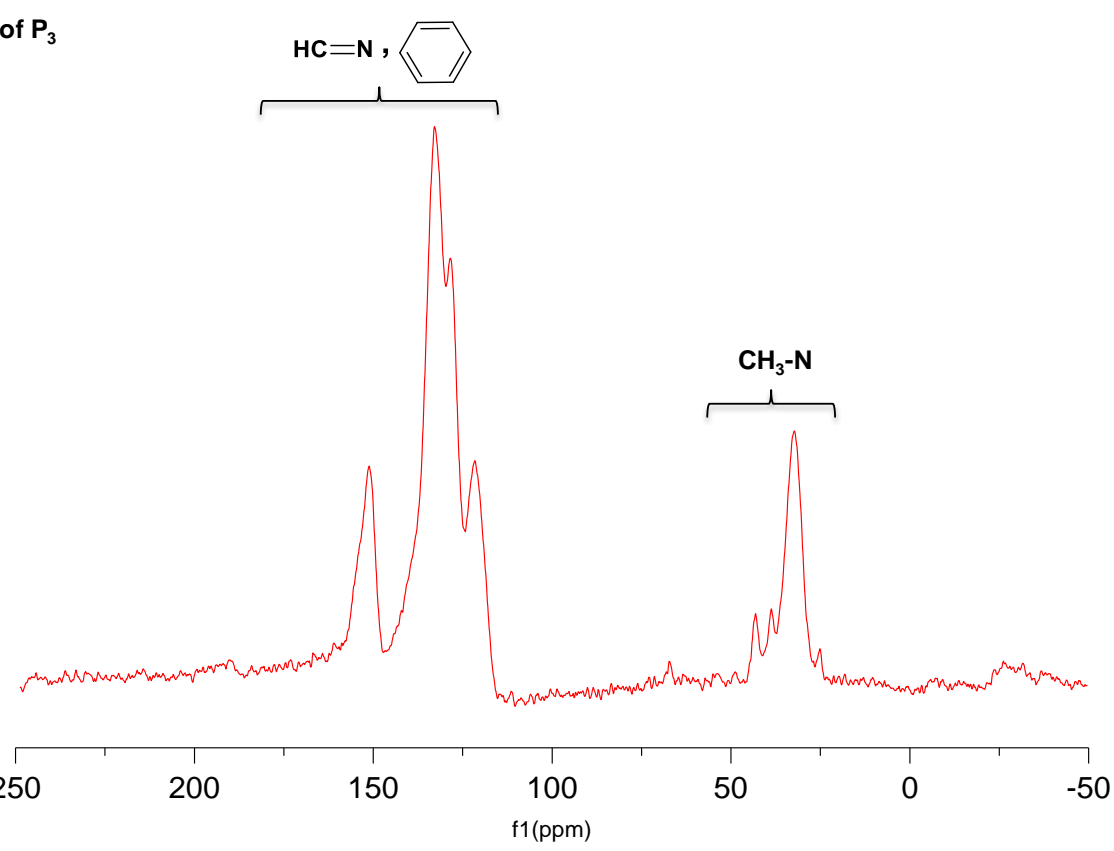


${ }^{13} \mathrm{C}$ NMR of $\mathrm{P}_{4}$
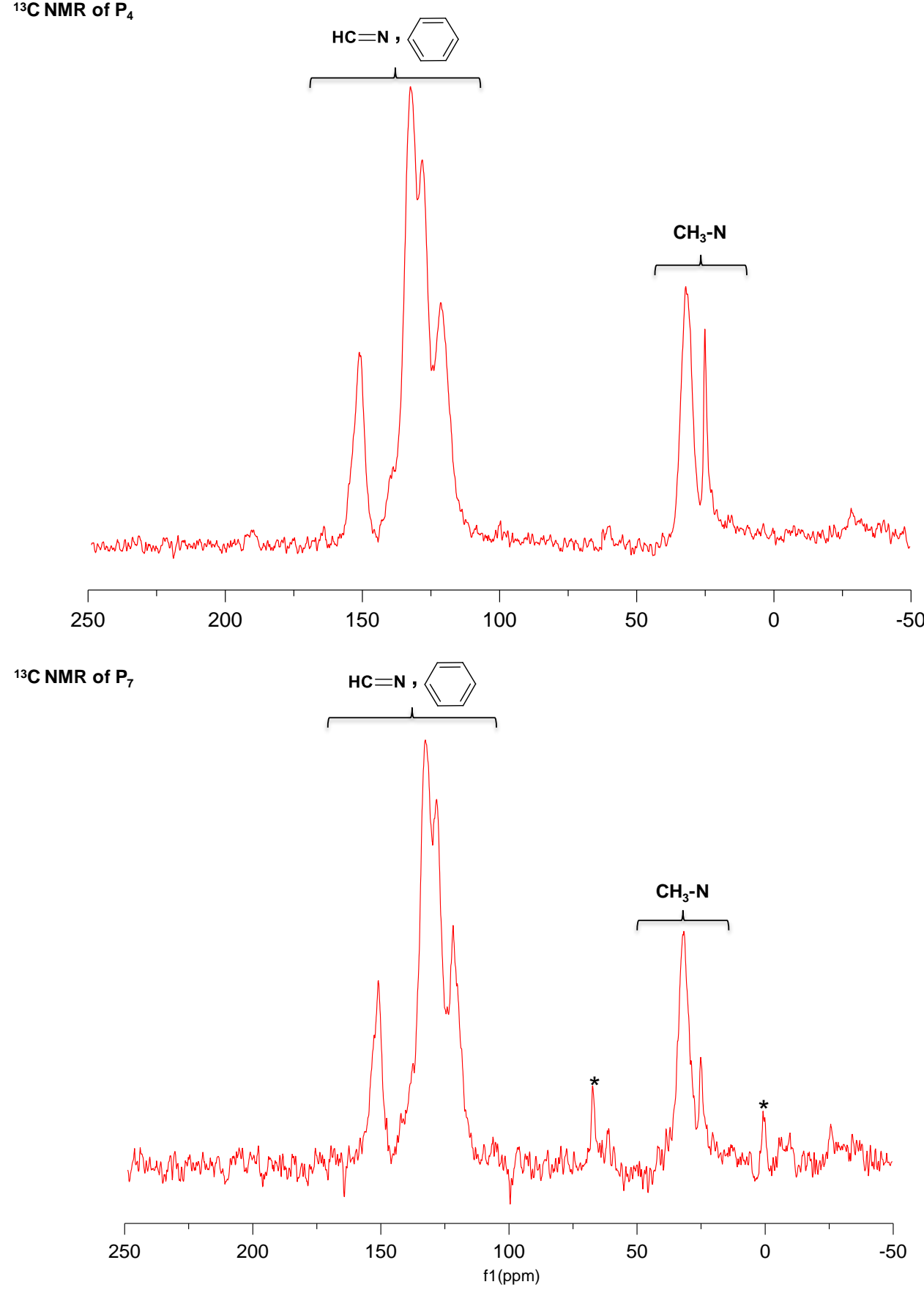
${ }^{13} \mathrm{C}$ NMR of $\mathrm{P}_{9}$

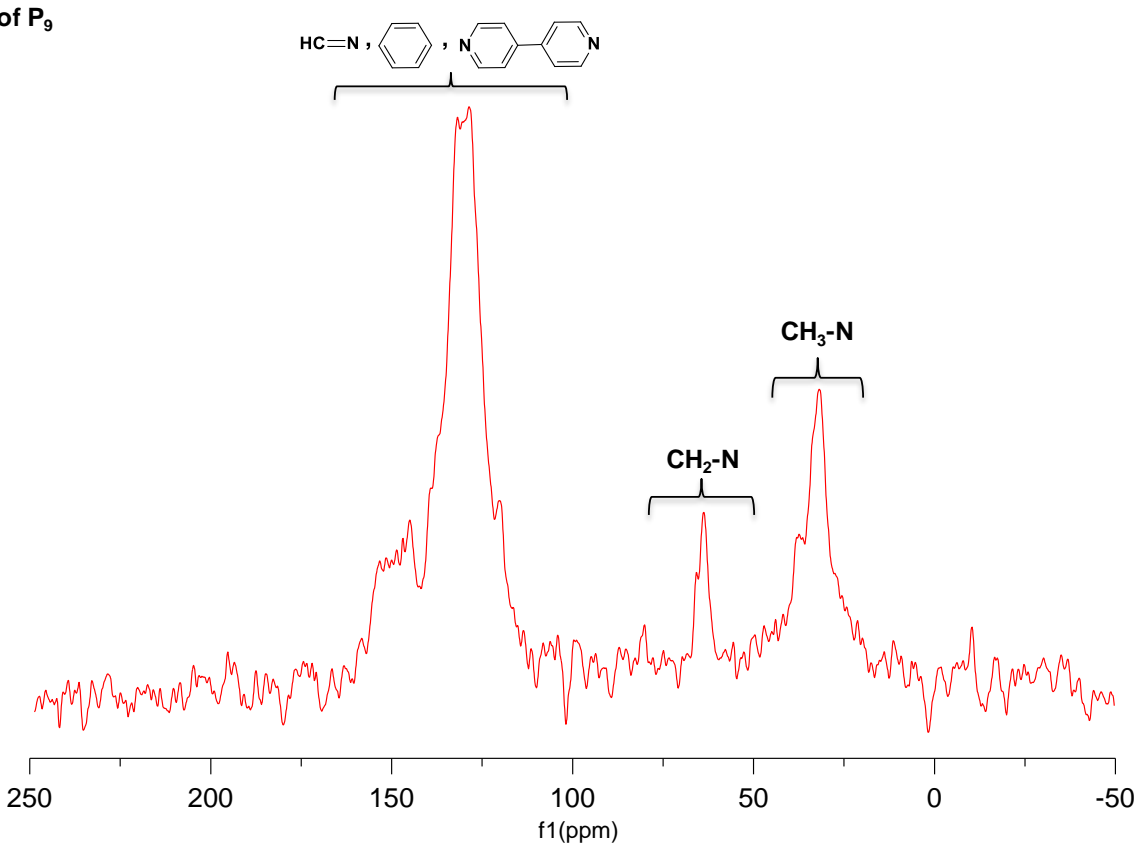

${ }^{13} \mathrm{C}$ NMR of $P_{10}$

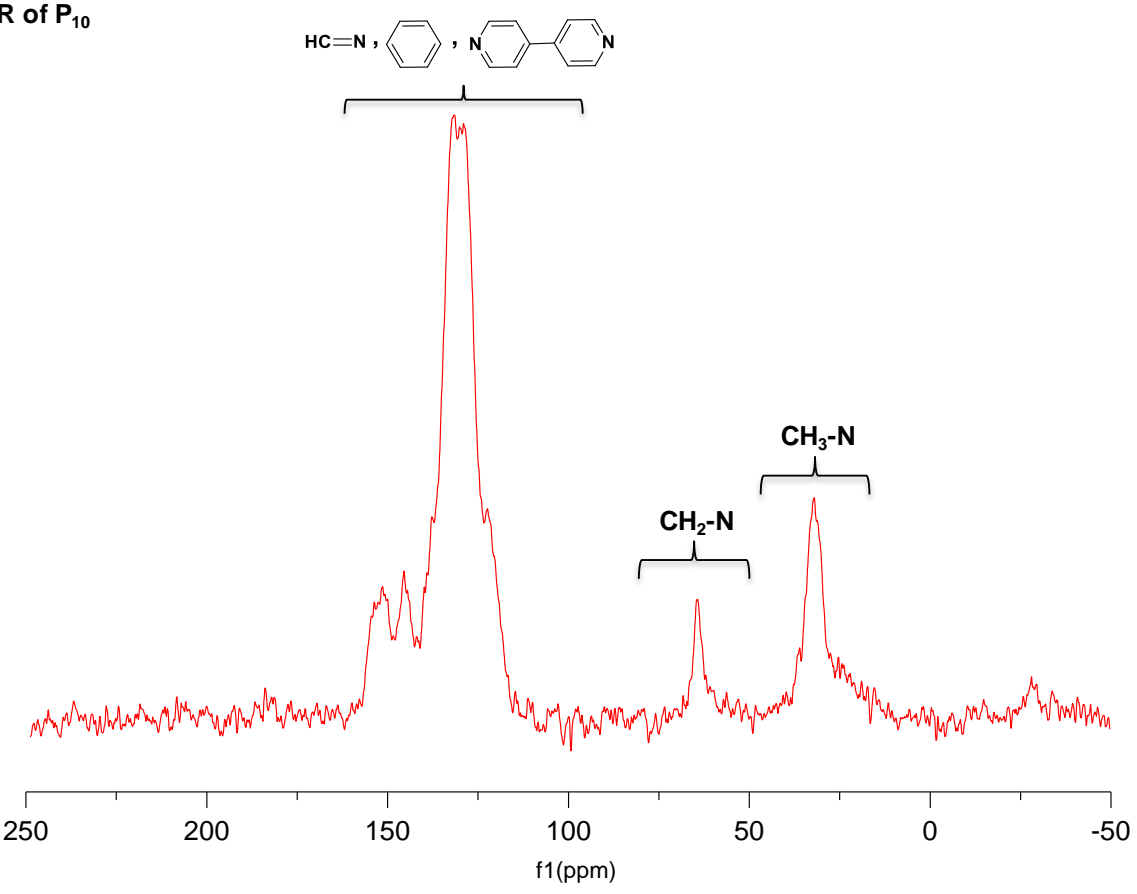


${ }^{13} \mathrm{C}$ NMR of $P_{11}$

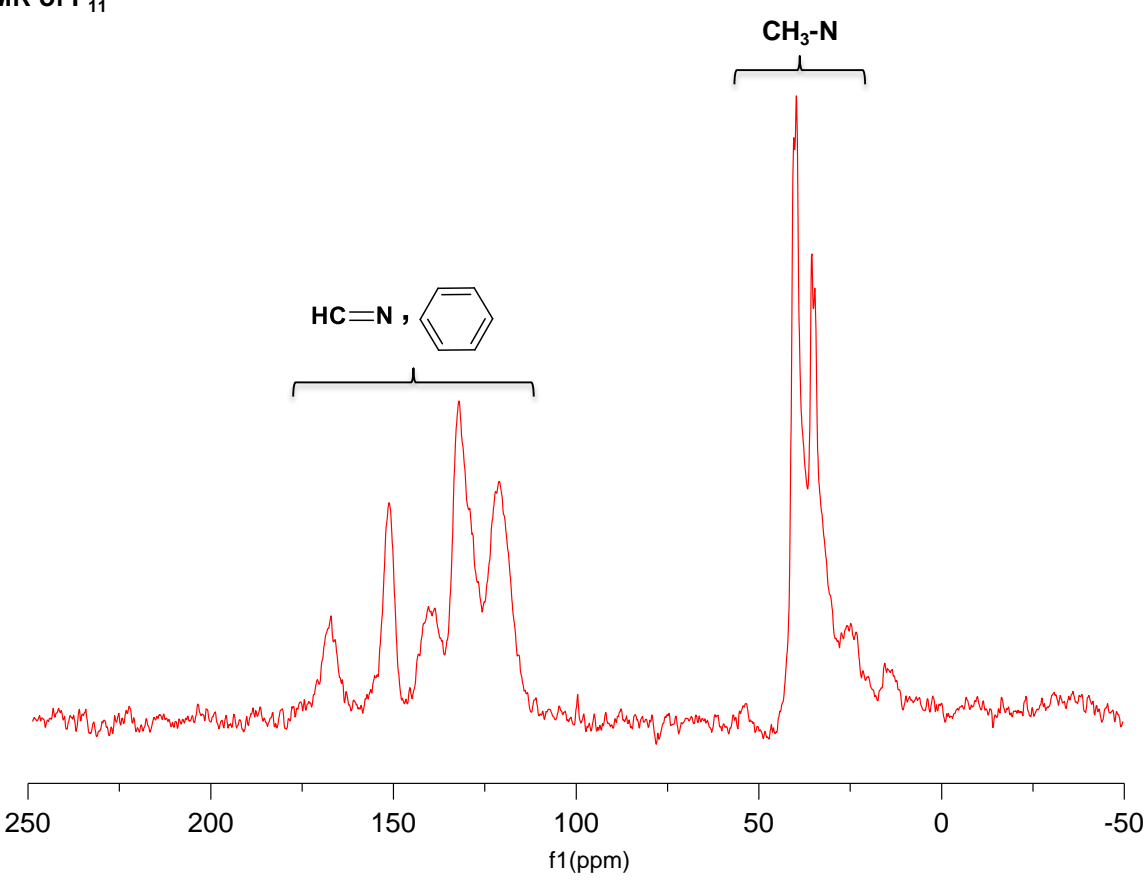


S4. ${ }^{31}$ P CP MAS NMR spectra

${ }^{31} \mathrm{P}$ NMR of $\mathrm{P}_{3}$

$8.30\left(\mathrm{PPh}_{2}\right)$

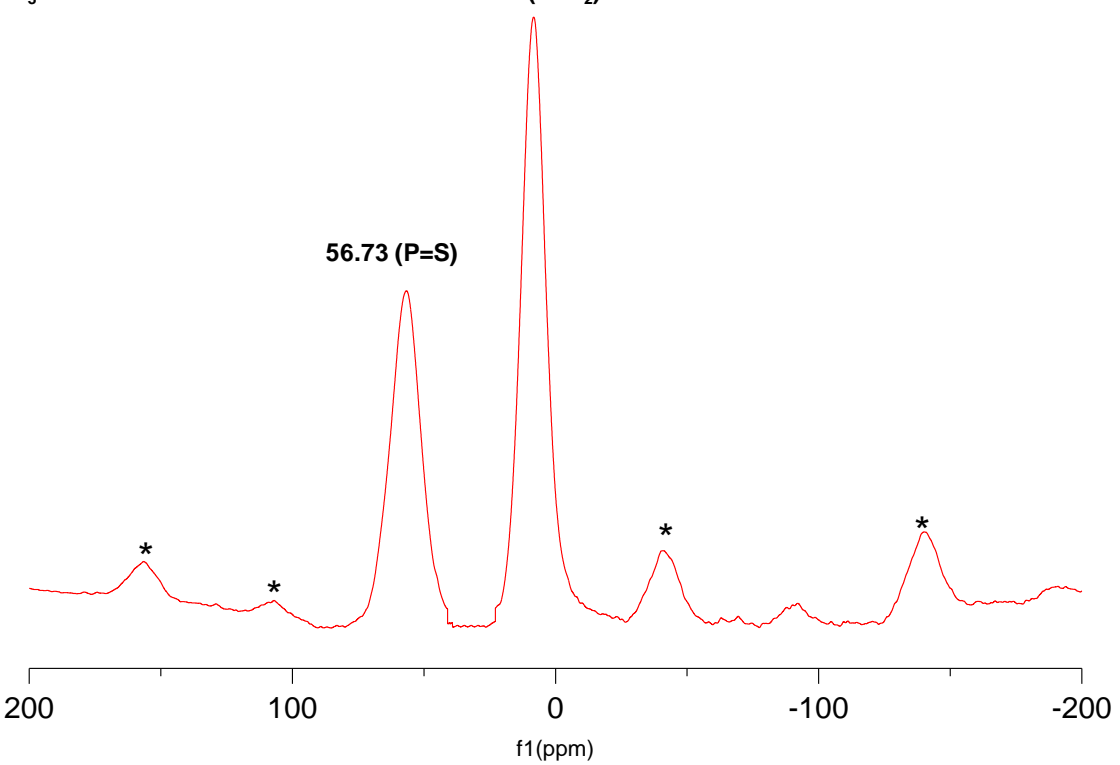

${ }^{31} \mathrm{P}$ NMR of $\mathbf{P}_{4}$

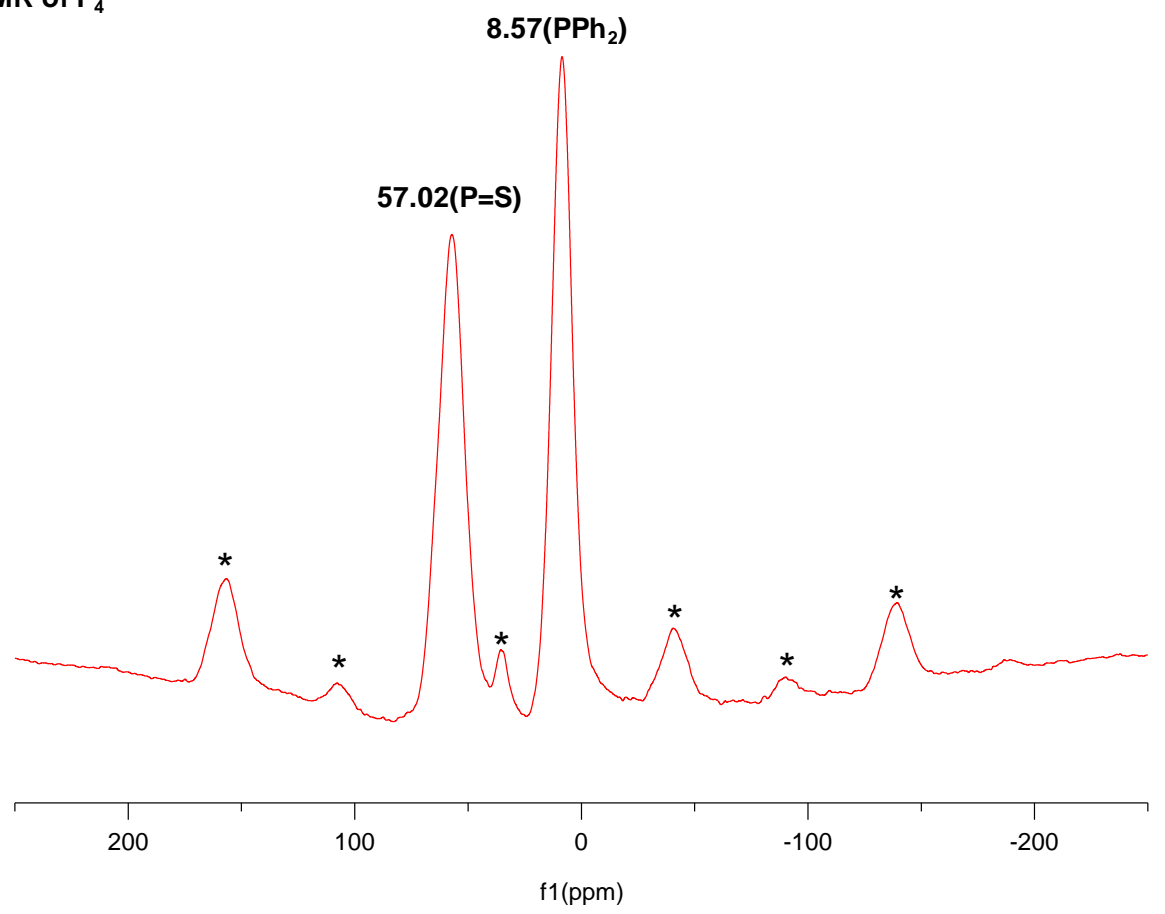


${ }^{31} \mathrm{P}$ NMR of $P_{5}$

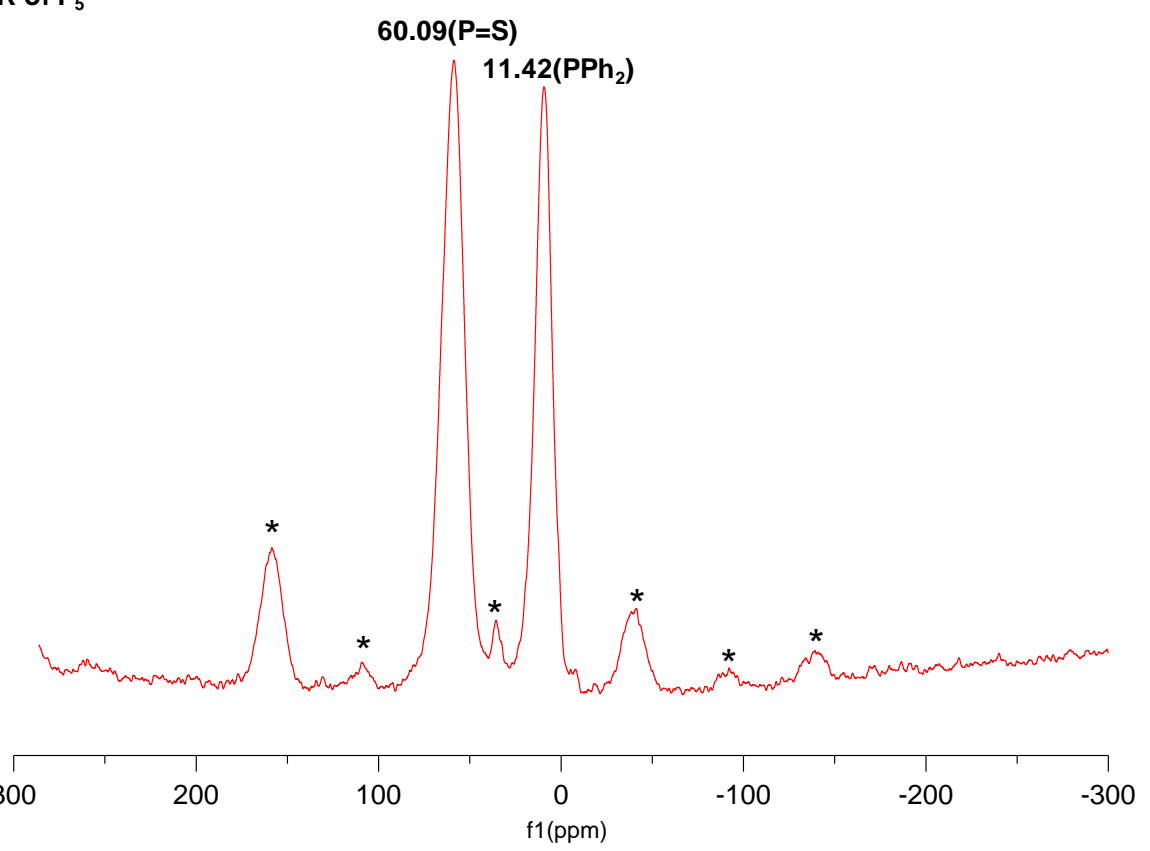

${ }^{31} \mathrm{P}$ NMR of $\mathrm{P}_{7}$

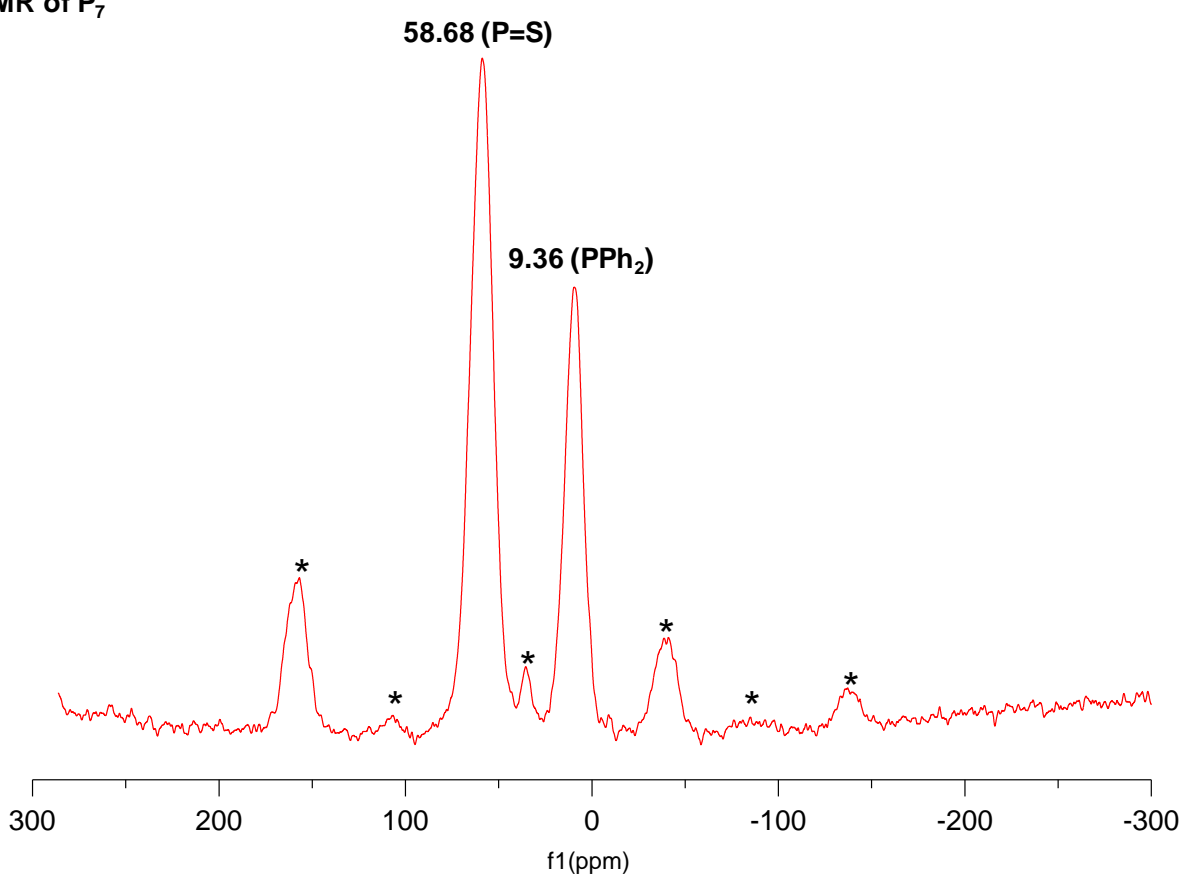




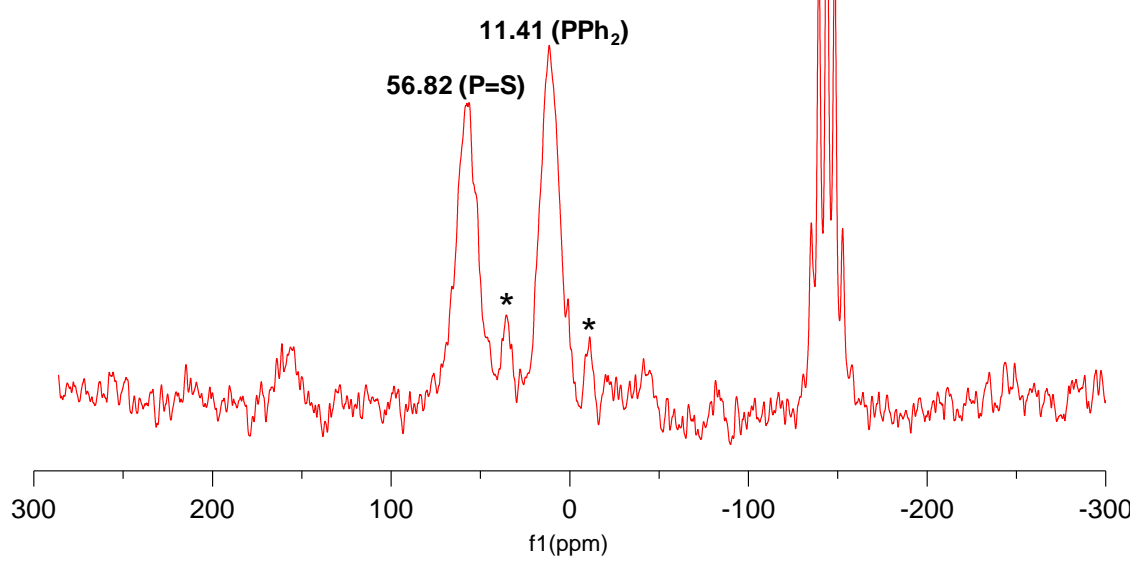

${ }^{31} \mathrm{P} N M R$ of $\mathrm{P}_{10}$

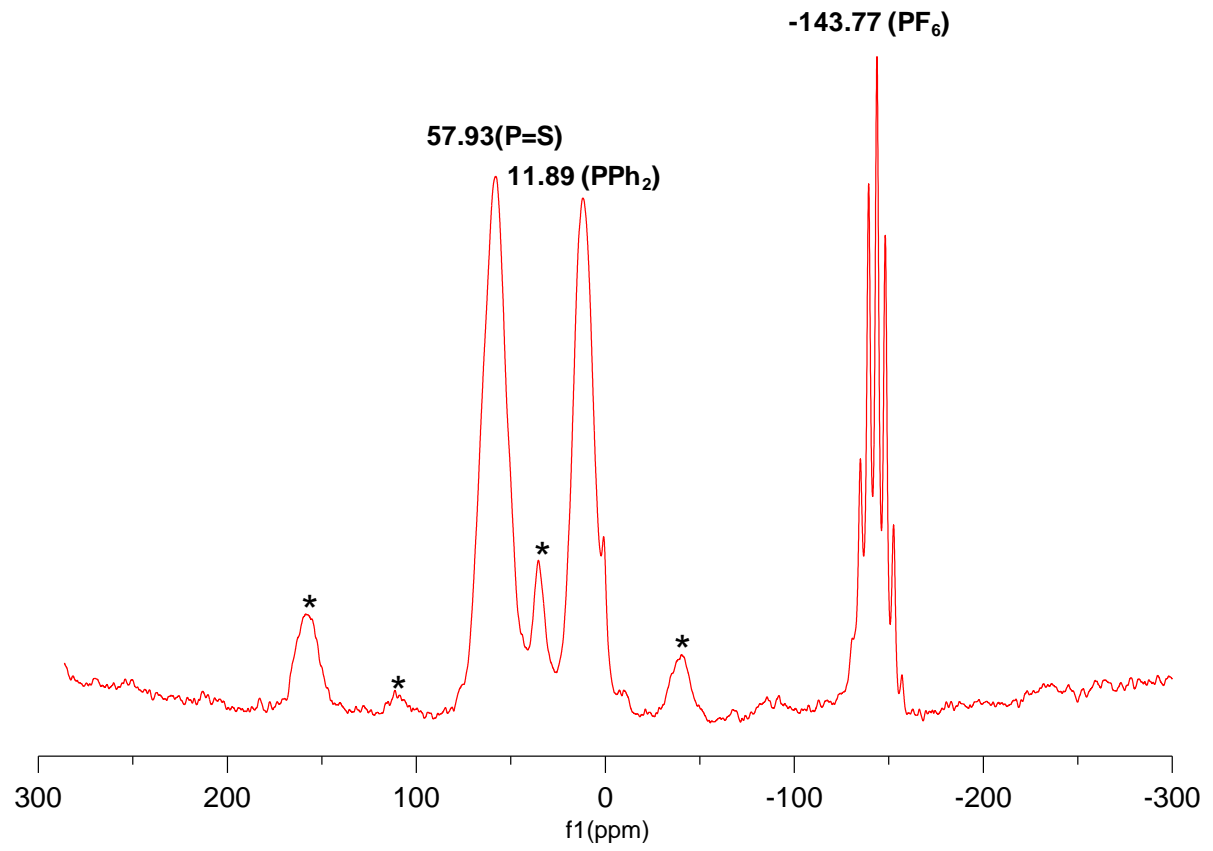


${ }^{31} \mathrm{P}$ NMR of $\mathrm{P}_{11}$

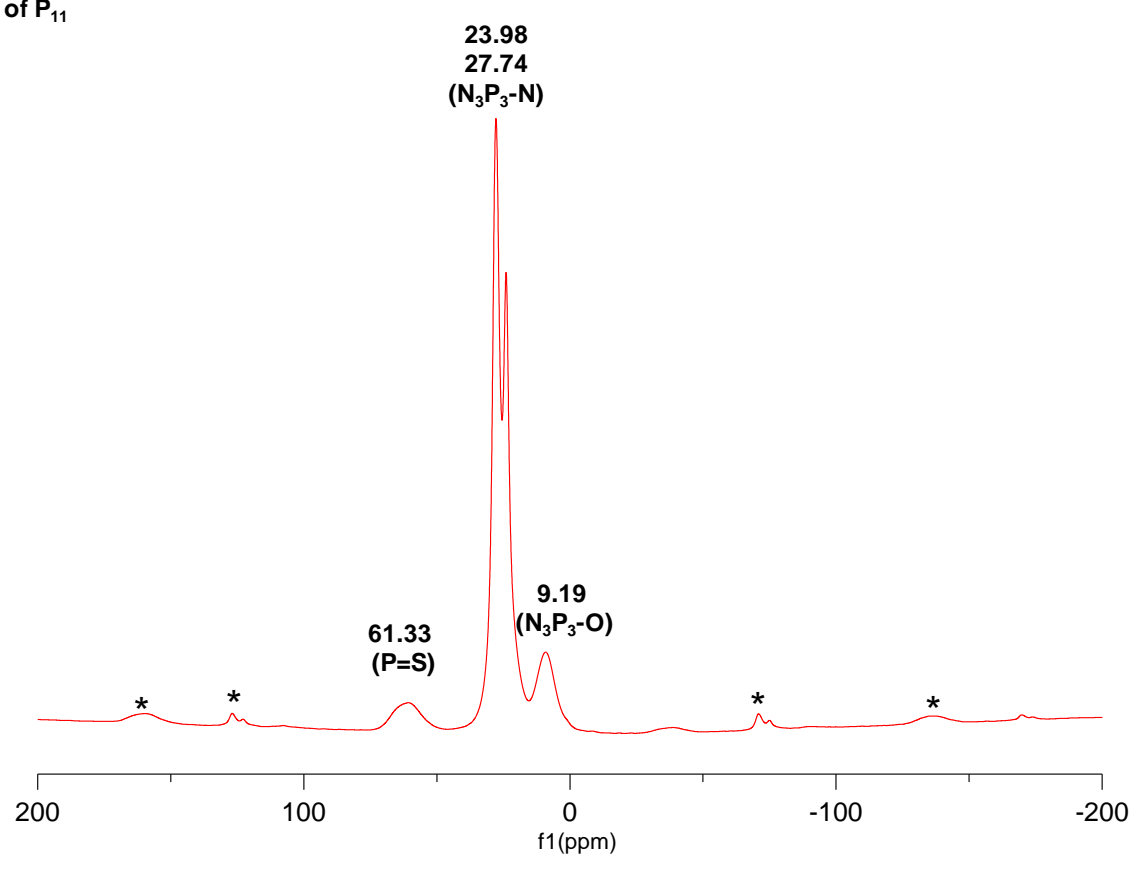


S5. DRUV spectra

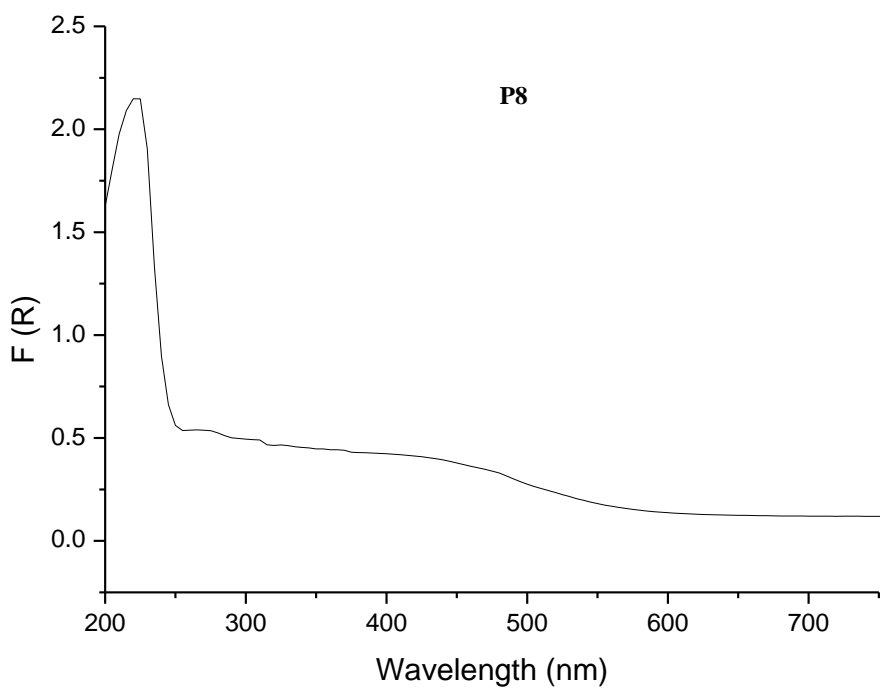


S6. SEM analysis

SEM of Polymer $\mathbf{P}_{1}$
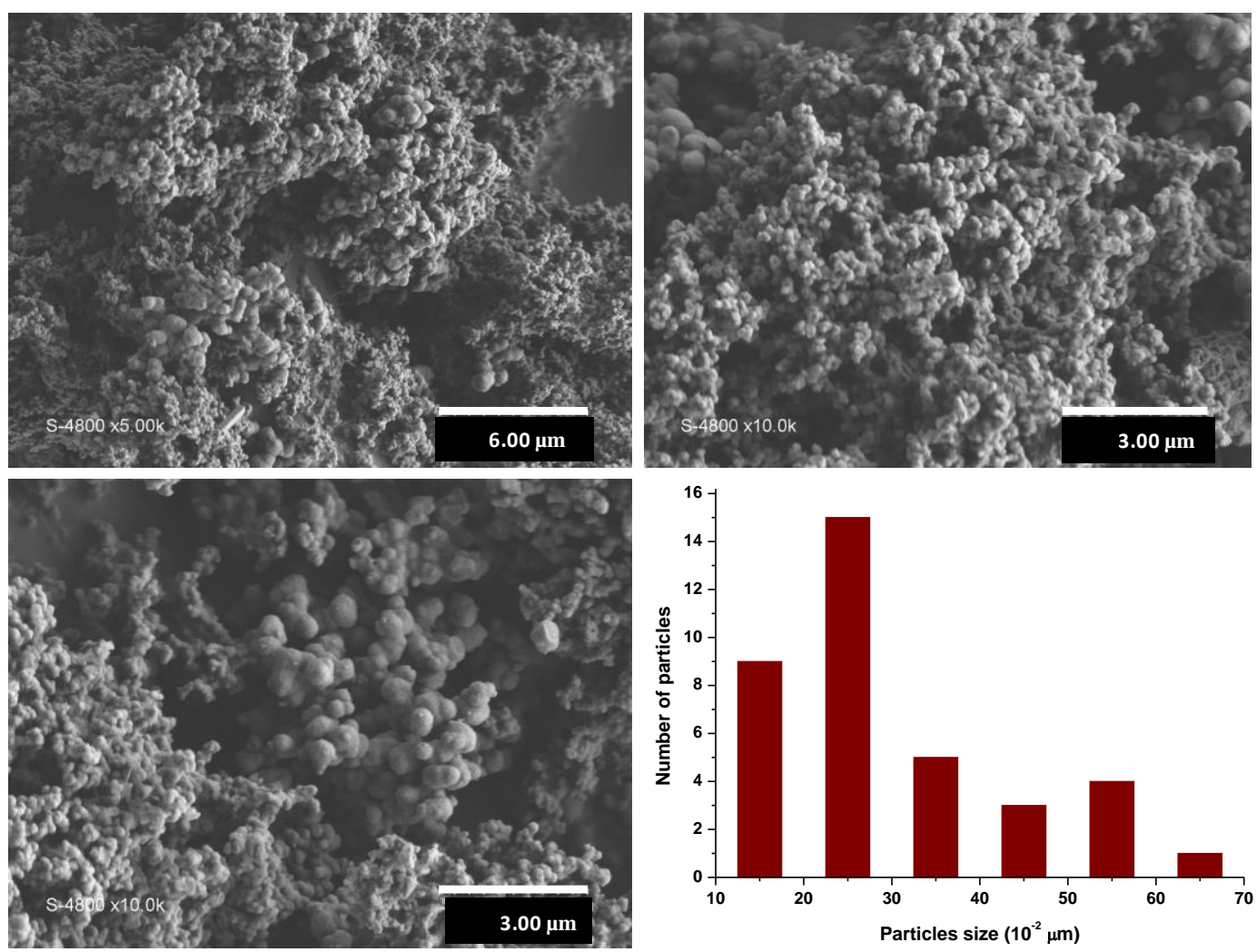

\section{SEM of Polymer $\mathrm{P}_{2}$}
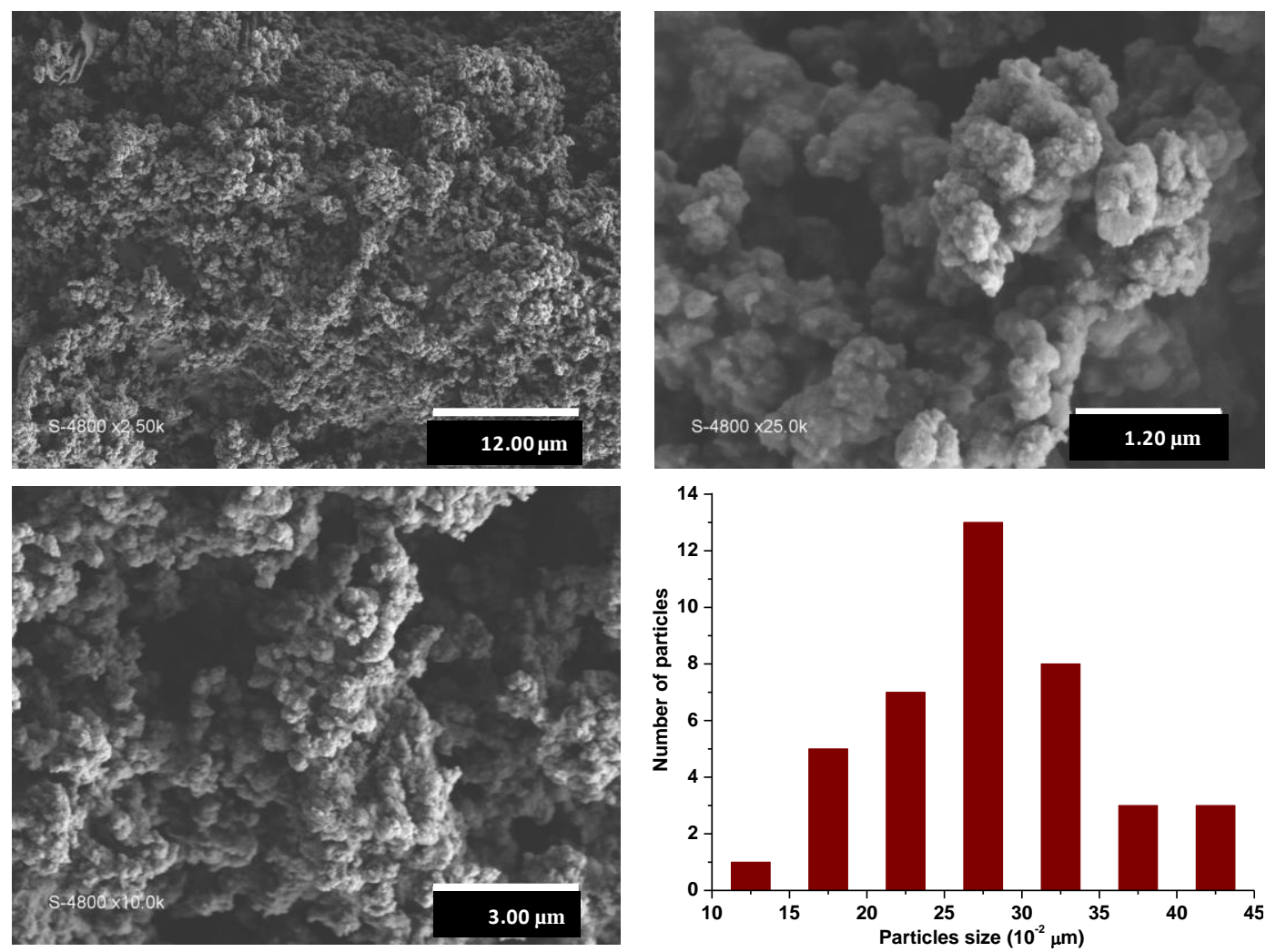
SEM of Polymer $\mathrm{P}_{6}$
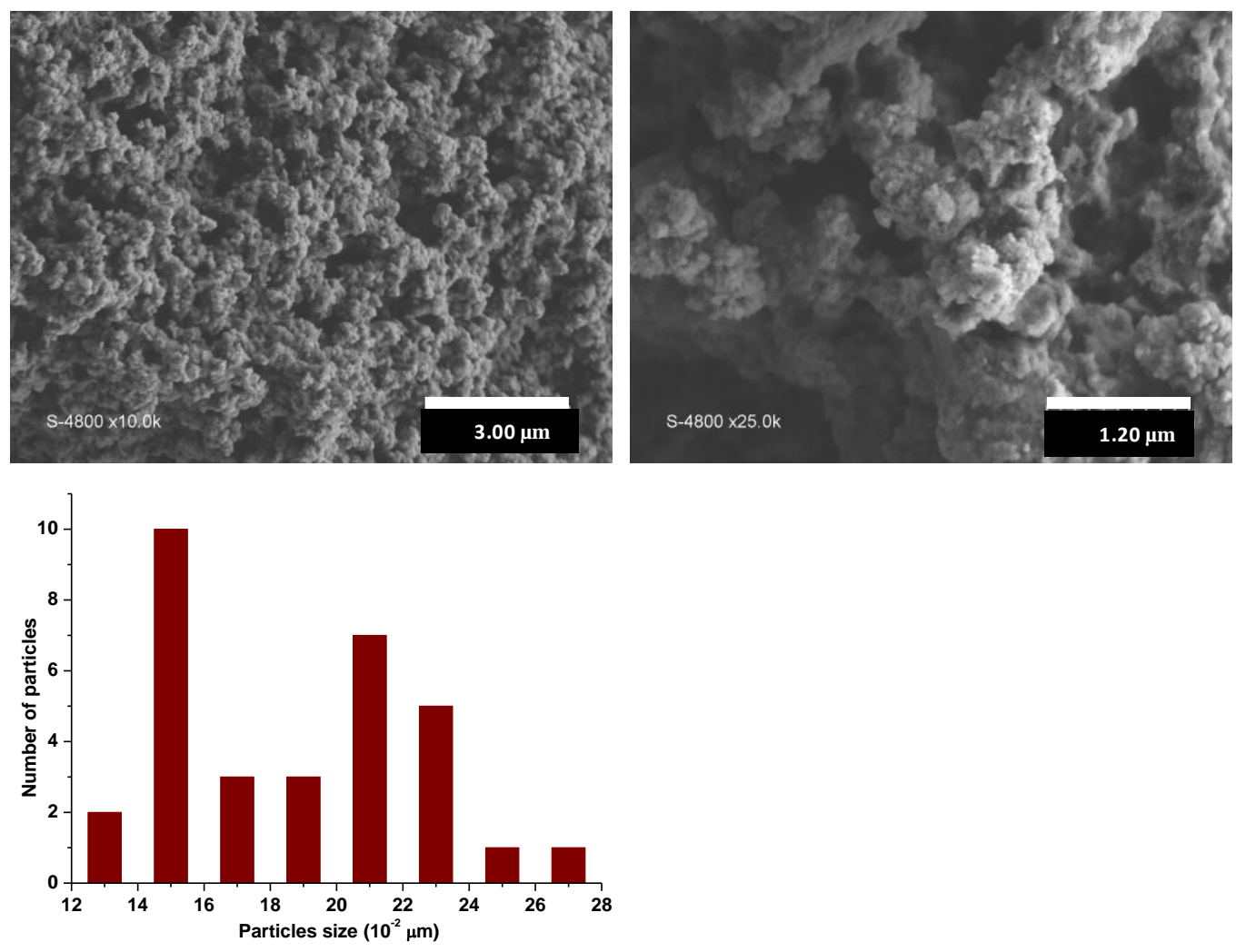

SEM of Polymer $\mathrm{P}_{7}$
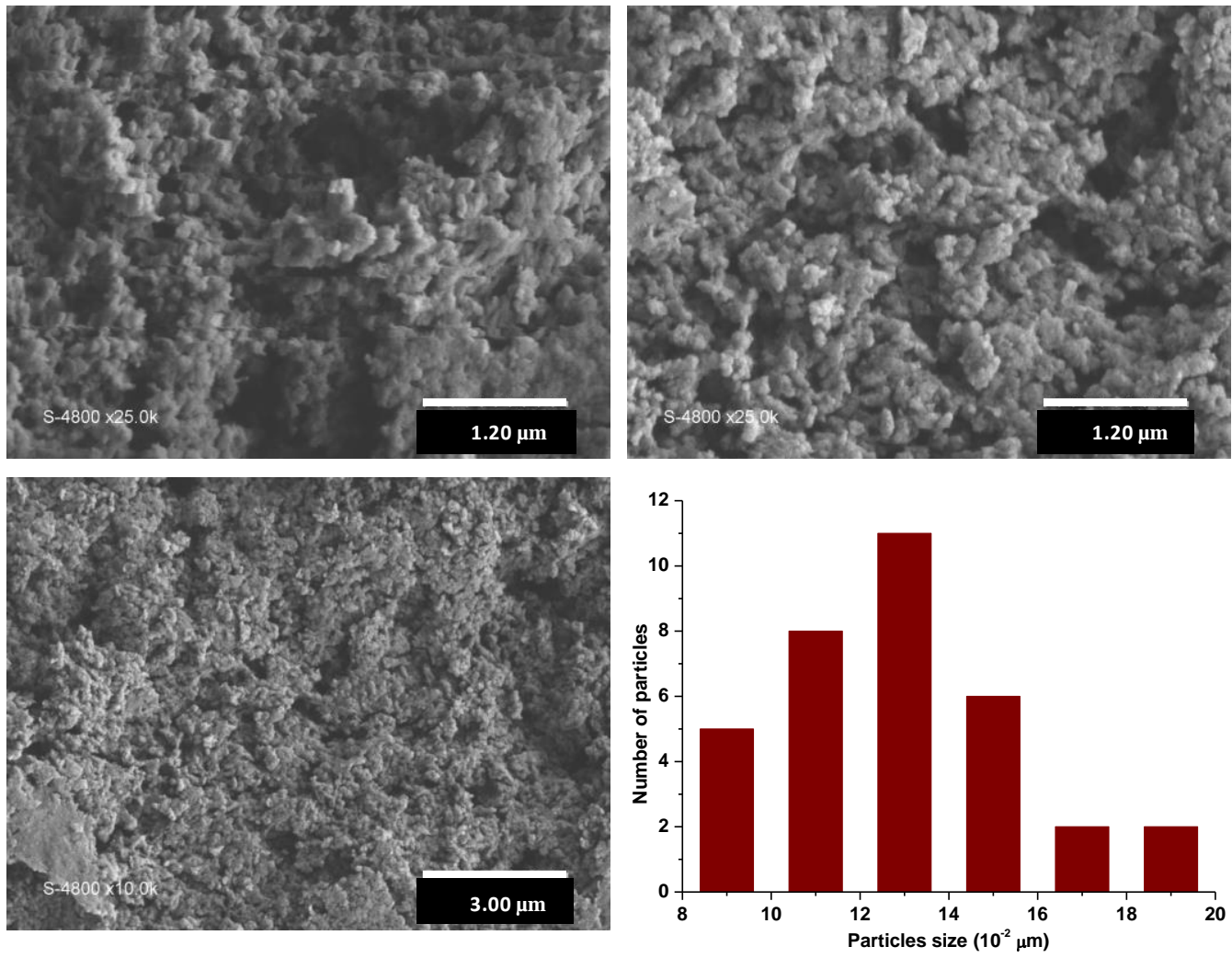
SEM of Polymer $\mathbf{P}_{11}$
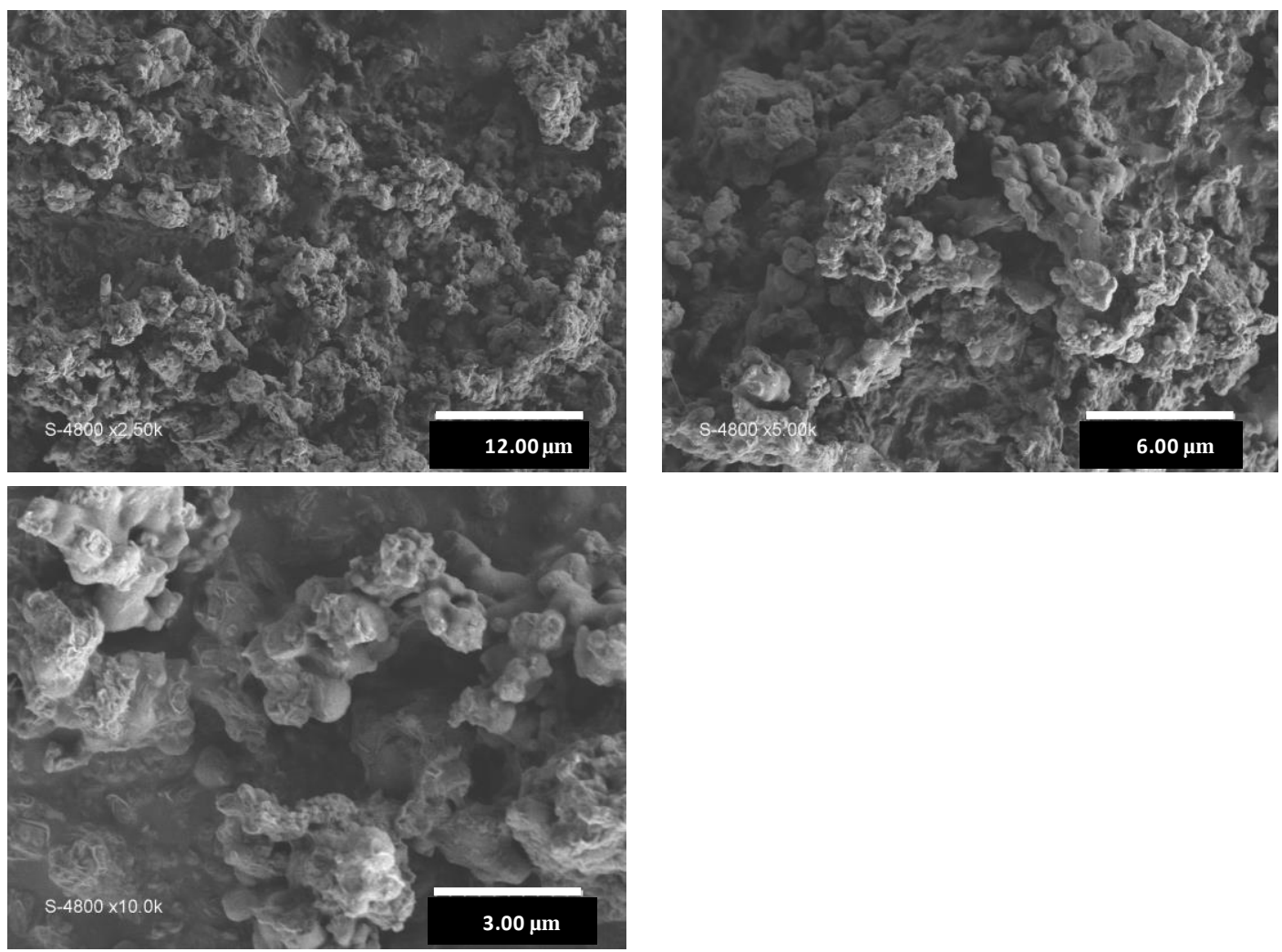


\section{S7. Nitrogen sorption analysis of polymers}
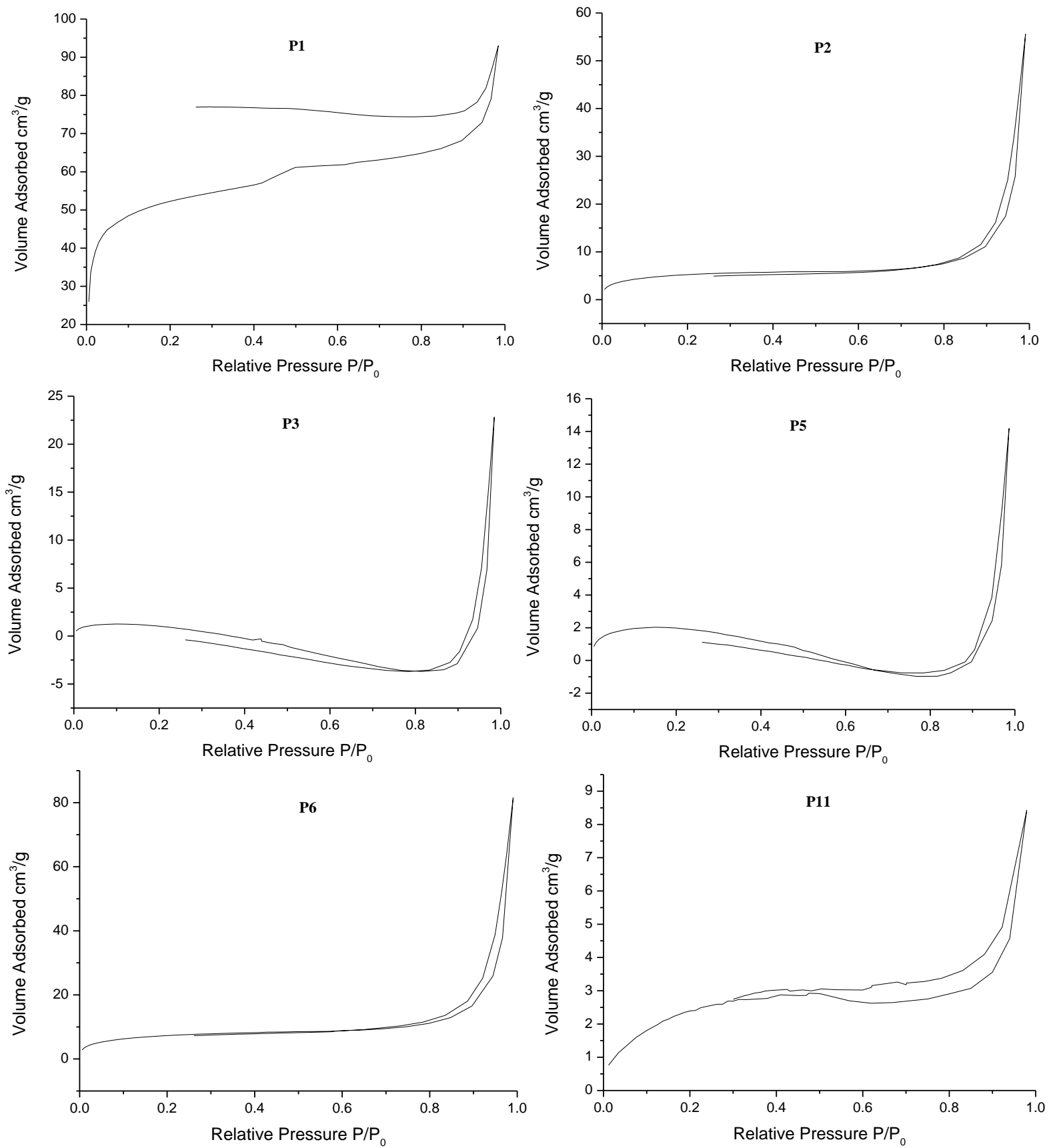


\section{S8. DRX analysis}
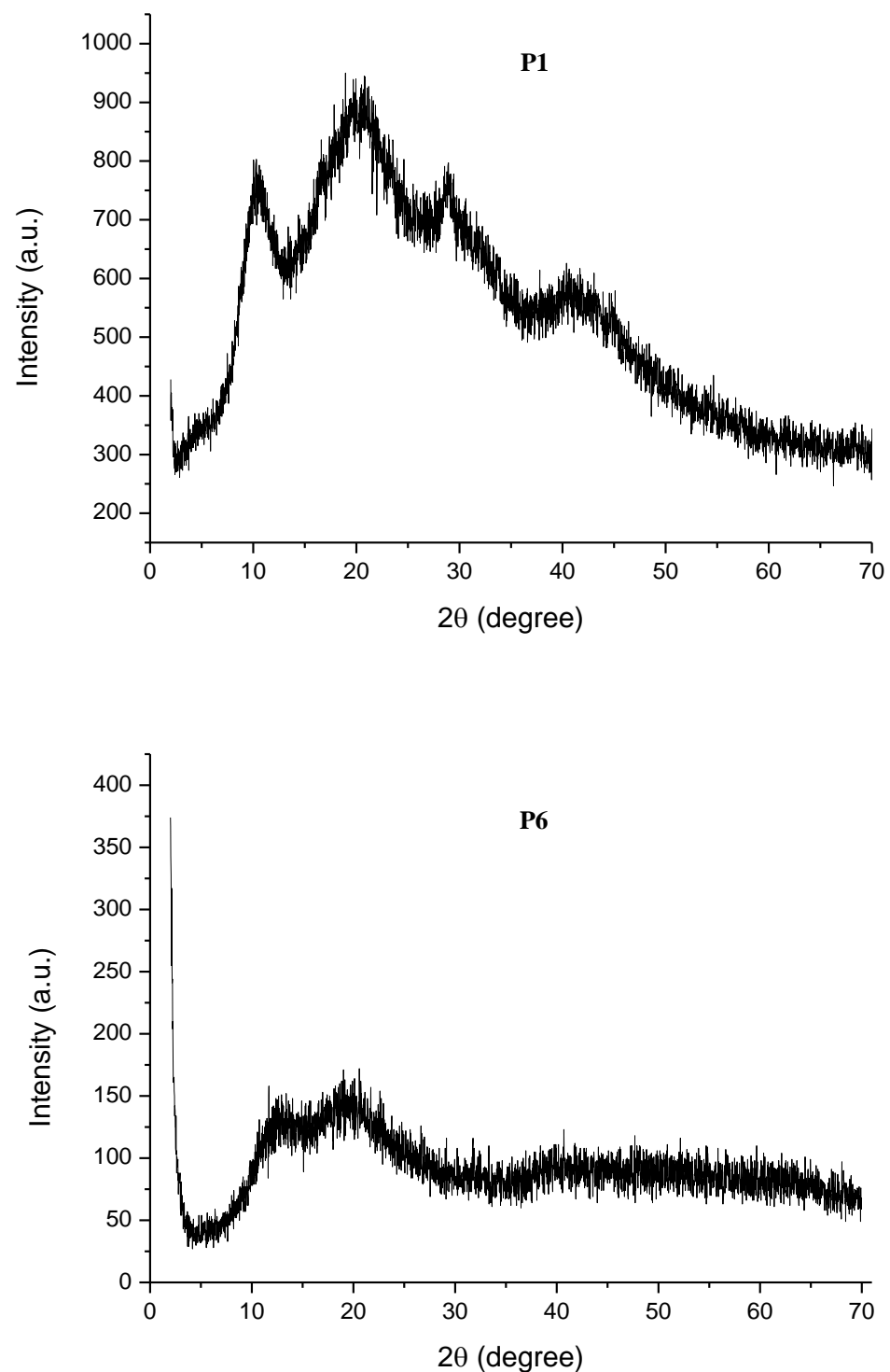


\section{S9. TEM of polymers}

S9a. TEM of polymer $P_{3}$ and $P_{5}$

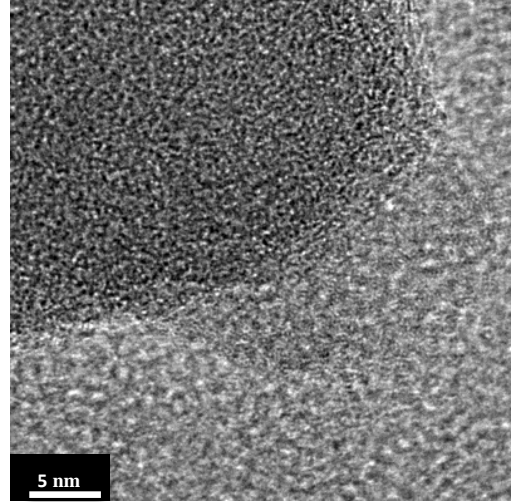

$\mathbf{P}_{3}$

S9b. TEM of polymer $P_{3}$ and $P_{5}$

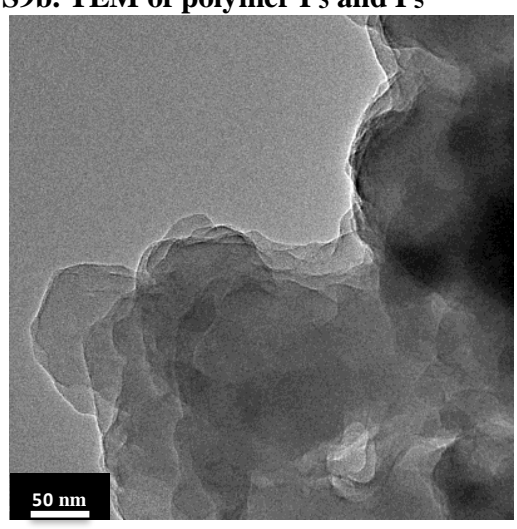

$\mathbf{P}_{3}$

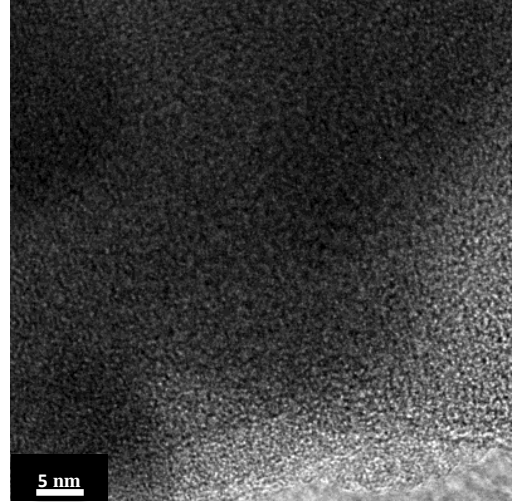

$\mathbf{P}_{5}$

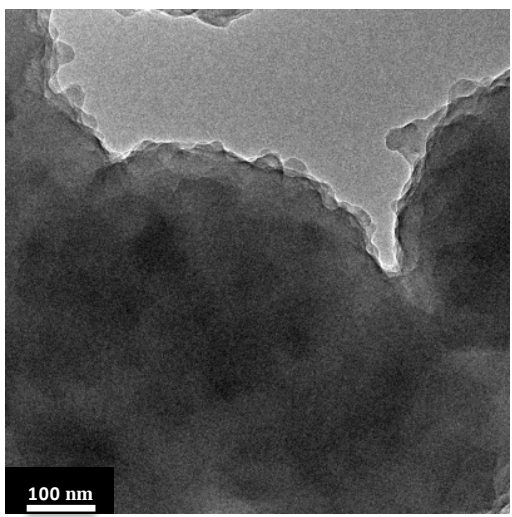

$\mathbf{P}_{5}$

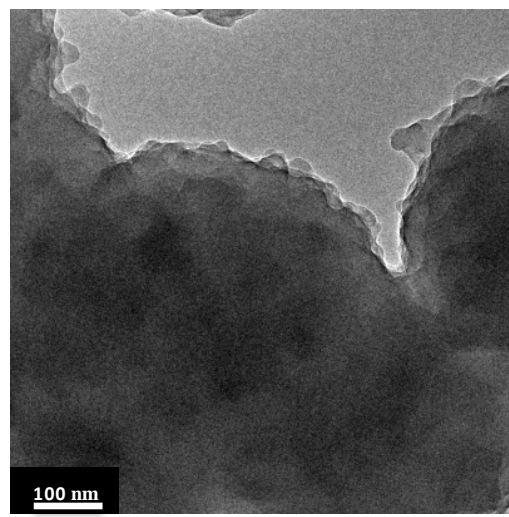

$P_{5}$

S9c. TEM of polymer $P_{3}$ and $P_{5}$

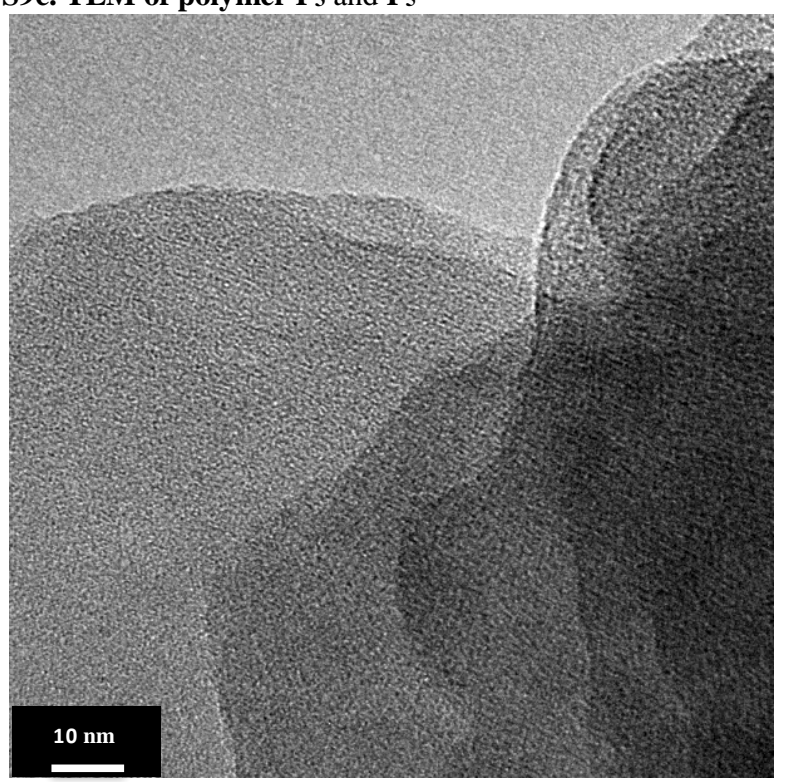

$\mathbf{P}_{3}$

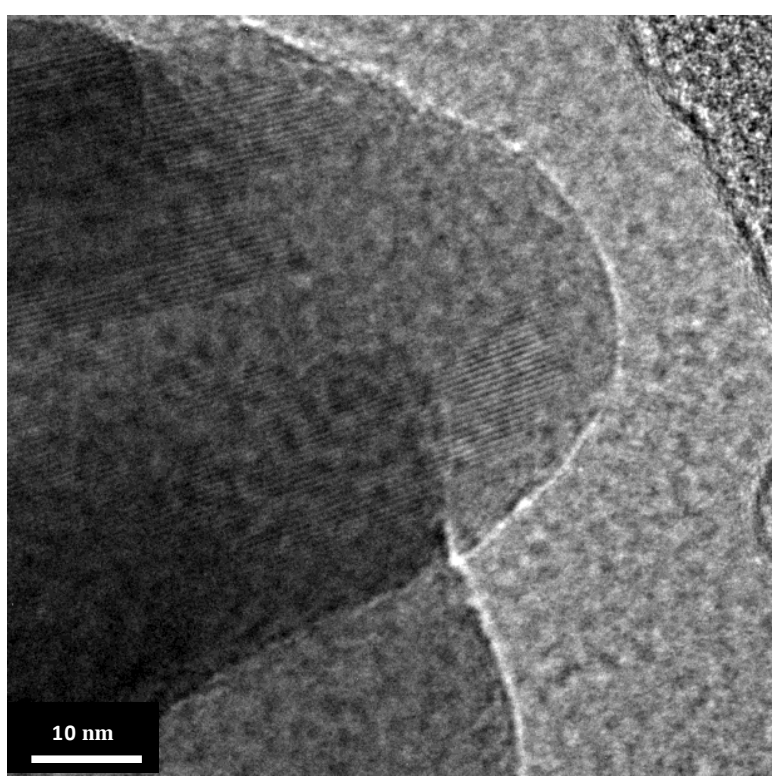

$\mathbf{P}_{5}$ 